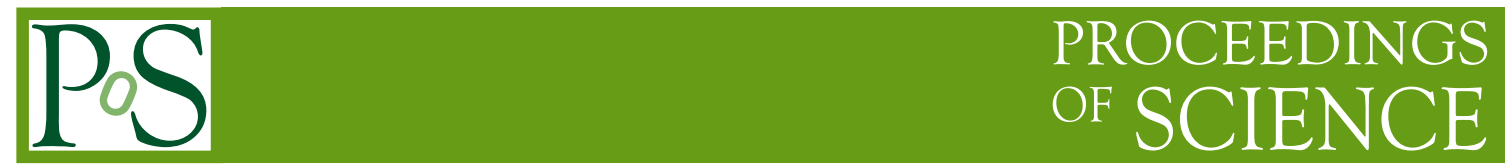

\title{
INTRODUCTION TO SUPERSYMMETRY
}

\section{Dmitri Kazakov}

Bogoliubov Laboratory of Theoretical Physics, JINR, Dubna, Russia

and

Institute for Theoretical and Experimental Physics, Moscow, Russia

E-mail: kazakovd@theor.jinr.ru

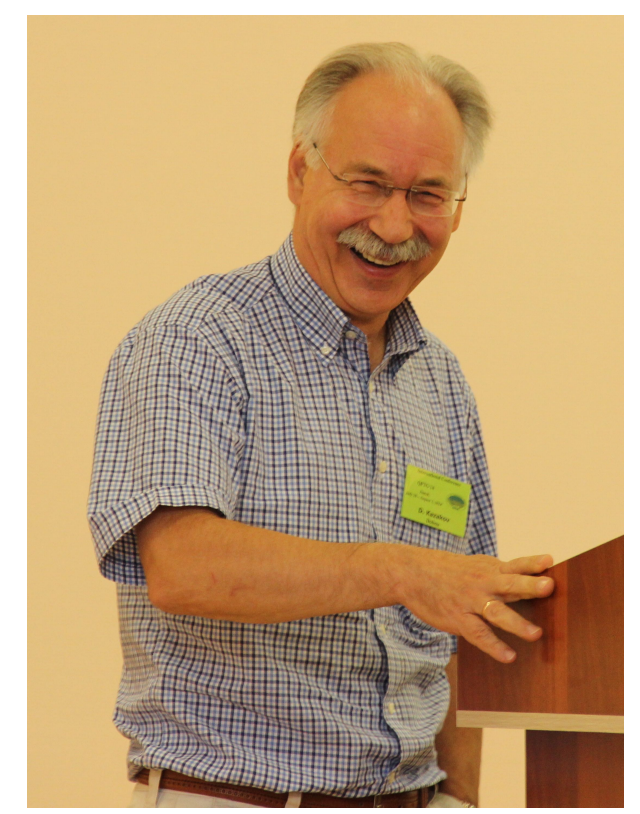

\begin{abstract}
Supersymmetry, a new symmetry that relates bosons and fermions in particle physics, still escapes observation. Search for supersymmetry is one of the main aims of the Large Hadron Collider. The present lectures contain a brief introduction to supersymmetry in particle physics. The main notions of supersymmetry are introduced. The supersymmetric extension of the Standard Model - the Minimal Supersymmetric Standard Model - is considered in more detail. Phenomenological features of the Minimal Supersymmetric Standard Model as well as possible experimental signatures of supersymmetry at the Large Hadron Collider are described.
\end{abstract}

Proceedings of the Corfu Summer Institute 2014 "School and Workshops on Elementary Particle Physics and Gravity", 3-21 September 2014, Corfu, Greece 


\section{Part I: Basics of Supersymmetry}

\section{What is supersymmetry}

Supersymmetry is a boson-fermion symmetry that is aimed to unify all forces in Nature including gravity within a singe framework $[1,2,3,4,5]$. Modern views on supersymmetry in particle physics are based on a string paradigm, though low energy manifestations of supersymmetry (SUSY) can be possibly found at modern colliders and in non-accelerator experiments.

Supersymmetry emerged from attempts to generalize the Poincaré algebra to mix representations with different spin [1]. It happened to be a problematic task due to "no-go" theorem preventing such generalizations [6]. The way out was found by introducing so-called graded Lie algebras, i. e. adding anti-commutators to usual commutators of the Lorentz algebra. Such a generalization, described below, appeared to be the only possible one within the relativistic field theory.

If $Q$ is a generator of the SUSY algebra, then acting on a boson state it produces a fermion one and vice versa

$$
\bar{Q} \mid \text { boson }\rangle=\mid \text { fermion }\rangle, Q \mid \text { fermion }\rangle=\mid \text { boson }\rangle .
$$

Since the bosons commute with each other and the fermions anticommute, one immediately finds that the SUSY generators should also anticommute, they must be fermionic, i. e. they must change the spin by a half-odd amount and change the statistics. The key element of the SUSY algebra is

$$
\left\{Q_{\alpha}, \bar{Q}_{\dot{\alpha}}\right\}=2 \sigma_{\alpha \dot{\alpha}}^{\mu} P_{\mu}
$$

where $Q$ and $\bar{Q}$ are the generators of the supersymmetry transformation and $P_{\mu}$ is the generator of translation, the four-momentum.

In what follows we describe the SUSY algebra in more detail and construct its representations which are needed to build the SUSY generalization of the Standard Model (SM) of fundamental interactions. Such a generalization is based on a softly broken SUSY quantum filed theory and contains the SM as the low energy theory.

Supersymmetry promises to solve some problems of the Standard Model and of Grand Unified Theories. In what follows we describe supersymmetry as the nearest option for the new physics on the TeV scale.

\section{Algebra of SUSY}

Consider the usual Poincaré algebra

$$
\begin{aligned}
& {\left[P_{\mu}, P_{v}\right]=0,} \\
& {\left[P_{\mu}, M_{\rho \sigma}\right]=i\left(g_{\mu \rho} P_{\sigma}-g_{\mu \sigma} P_{\rho}\right),} \\
& {\left[M_{\mu v}, M_{\rho \sigma}\right]=i\left(g_{v \rho} M_{\mu \sigma}-g_{v \sigma} M_{\mu \rho}-g_{\mu \rho} M_{v \sigma}+g_{\mu \sigma} M_{v \rho}\right),}
\end{aligned}
$$

where $P_{\mu}$ and $M_{\mu \nu}$ are the four-momentum and angular four momentum, respectively. The SuperPoincaré Lie algebra contains additional SUSY generators $Q_{\alpha}^{i}$ and $\bar{Q}_{\dot{\alpha}}^{i}$ [3]. On general grounds 
they might obey the following set of commutator or anti-commutator Lorentz invariant relations:

$$
\begin{aligned}
& {\left[P_{\mu}, Q_{\alpha}^{i}\right]=c_{1}\left(\sigma_{\mu}\right)_{\alpha \dot{\alpha}} \bar{Q}^{\dot{\alpha} i}} \\
& {\left[P_{\mu}, \bar{Q}_{\dot{\alpha}}^{i}\right]=c_{2}\left(\tilde{\sigma}_{\mu}\right)_{\dot{\alpha} \alpha} Q^{\alpha i}} \\
& {\left[M_{\mu v}, Q_{\alpha}^{i}\right]=c_{3}\left(\sigma_{\mu v}\right)_{\alpha}^{\beta} \bar{Q}_{\beta}^{i},} \\
& {\left[M_{\mu v}, \bar{Q}_{\dot{\alpha}}^{i}\right]=c_{4}\left(\tilde{\sigma}_{\mu v}\right)_{\dot{\alpha}}^{\dot{\beta}} Q_{\dot{\beta}}^{i},} \\
& \left\{Q_{\alpha}^{i}, Q_{\beta}^{j}\right\}=c_{5} \varepsilon_{\alpha \beta} Z^{i j}+\tilde{c}_{5}\left(\sigma^{\mu v}\right)_{\alpha \beta} M_{\mu v} X^{i j} \\
& \left\{\bar{Q}_{\dot{\alpha}}^{i}, \bar{Q}_{\dot{\beta}}^{j}\right\}=c_{6} \varepsilon_{\dot{\alpha} \dot{\beta}} \bar{Z}^{i j}+\tilde{c}_{6}\left(\tilde{\sigma}^{\mu v}\right)_{\dot{\alpha} \dot{\beta}} M_{\mu v} \bar{X}^{i j} \\
& \left\{Q_{\alpha}^{i}, \bar{Q}_{\dot{\alpha}}^{j}\right\}=2 c_{7}\left(\sigma^{\mu}\right)_{\alpha \dot{\alpha}} P_{\mu} \delta^{i j},
\end{aligned}
$$

with arbitrary coefficients $c_{1}, \ldots, c_{7}$. However, to satisfy the algebra the operators have to obey the Jacobi identities

$$
\begin{aligned}
& {\left[B_{1},\left[B_{2}, B_{3}\right]\right]+\left[B_{2},\left[B_{3}, B_{1}\right]\right]+\left[B_{3},\left[B_{1}, B_{2}\right]\right]=0,} \\
& {\left[B,\left\{F_{1}, F_{2}\right\}\right]+\left\{F_{1},\left[F_{2}, B\right]\right\}-\left\{F_{2},\left[B, F_{1}\right]\right\}=0,} \\
& {\left[B_{1},\left[B_{2}, F\right]\right]+\left[B_{2},\left[F, B_{1}\right]\right]+\left[F,\left[B_{1}, B_{2}\right]\right]=0,} \\
& {\left[F_{1},\left\{F_{2}, F_{3}\right\}\right]+\left[F_{2},\left\{F_{3}, F_{1}\right\}\right]+\left[F_{3},\left\{F_{1}, F_{2}\right\}\right]=0,}
\end{aligned}
$$

where $B_{i}$ are bosonic and $F_{i}$ are fermionic generators. Applying Jacobi identities to Eqns.(2.2) one gets a unique solution

$$
c_{1}=c_{2}=\tilde{c}_{5}=\tilde{c}_{6}=0, c_{3}=c_{4}=i, c_{5}=c_{6}=c_{7}=1, X^{i j}=\bar{X}^{i j}=0 .
$$

This leads to the following Super-Poincaré Lie algebra

$$
\begin{aligned}
& {\left[P_{\mu}, P_{v}\right]=0,} \\
& {\left[P_{\mu}, M_{\rho \sigma}\right]=i\left(g_{\mu \rho} P_{\sigma}-g_{\mu \sigma} P_{\rho}\right),} \\
& {\left[M_{\mu v}, M_{\rho \sigma}\right]=i\left(g_{v \rho} M_{\mu \sigma}-g_{v \sigma} M_{\mu \rho}-g_{\mu \rho} M_{v \sigma}+g_{\mu \sigma} M_{v \rho}\right),} \\
& {\left[Q_{\alpha}^{i}, P_{\mu}\right]=\left[\bar{Q}_{\dot{\alpha}}^{i}, P_{\mu}\right]=0,} \\
& \left.\left[M_{\mu v}\right], Q_{\alpha}^{i}\right]=i\left(\sigma_{\mu v}\right)_{\alpha}^{\beta} Q_{\beta}^{i}, \quad\left[M_{\mu v}, \bar{Q}_{\dot{\alpha}}^{i}\right]=i\left(\bar{\sigma}_{\mu v}\right)_{\dot{\alpha}}^{\dot{\beta}} \bar{Q}_{\dot{\beta}}^{i}, \\
& \left\{Q_{\alpha}^{i}, \bar{Q}_{\dot{\beta}}^{j}\right\}=2 \delta^{i j}\left(\sigma^{\mu}\right)_{\alpha \dot{\beta}} P_{\mu}, \\
& \left\{Q_{\alpha}^{i}, Q_{\beta}^{j}\right\}=\varepsilon_{\alpha \beta} Z^{i j}, \\
& \left\{\bar{Q}_{\dot{\alpha}}^{i}, \bar{Q}_{\dot{\beta}}^{j}\right\}=\varepsilon_{\dot{\alpha} \dot{\beta}} \tilde{Z}^{i j}, \quad\left[Z_{i j}, \text { anything }\right]=0, \\
& \alpha, \dot{\alpha}=1,2 \quad i, j=1,2, \ldots, N .
\end{aligned}
$$

Here $Z_{i j}$ are the so-called central charges; $\alpha, \dot{\alpha}, \beta, \dot{\beta}$ are the spinorial indices.

In the simplest case one has one spinor generator $Q_{\alpha}$ (and the conjugated one $\bar{Q}_{\dot{\alpha}}$ ) that corresponds to the ordinary or $N=1$ supersymmetry. When $N>1$ one has the extended supersymmetry. The statement that the relations (2.4) represent the most general extension of the Poincare algebra (2.1) by means of fermionic generators is referred as the Haag - Lopuszanski- Sohnius theorem which was proved in 1975 [7]. 


\section{Representations of SUSY algebra}

A natural question arises: how many SUSY generators are possible, i. e. what is the value of $N$ ? To answer this question, consider massless states. Let us start with the ground state labeled by the energy and the helicity, i. e. the projection of the spin on the direction of momenta, and let it be annihilated by $Q_{i}$

$$
\text { Vacuum }=|E, \lambda\rangle, \quad Q_{i}|E, \lambda\rangle=0 .
$$

Then one- and many-particle states can be constructed with the help of creation operators as

\begin{tabular}{llc} 
State & Expression & \# of states \\
\cline { 3 - 3 } vacuum & $|E, \lambda\rangle$ & 1 \\
1-particle & $\bar{Q}_{i}|E, \lambda\rangle=\left|E, \lambda+\frac{1}{2}\right\rangle_{i}$ & $N$ \\
2-particle & $\left.\bar{Q}_{i} \bar{Q}_{j}|E, \lambda=| E, \lambda+1\right\rangle_{i j}$ & $\frac{N(N-1)}{2}$ \\
$\ldots$ & $\ldots$ & $\ldots$ \\
$N$-particle & $\bar{Q}_{1} \ldots \bar{Q}_{N}|E, \lambda\rangle=\left|E, \lambda+\frac{N}{2}\right\rangle$ & 1
\end{tabular}

The total \# of states is: $\sum_{k=0}^{N}\left(\begin{array}{c}N \\ k\end{array}\right)=2^{N}=2^{N-1}$ bosons $+2^{N-1}$ fermions.

The energy $E$ is not changed, since according to (2.4) the operators $\bar{Q}_{i}$ commute with the Hamiltonian.

Thus, one has a sequence of bosonic and fermionic states and the total number of the bosons equals to that of the fermions. This is a generic property of any supersymmetric theory. However, in CPT invariant theories the number of states is doubled, since CPT transformation changes the sign of the helicity. Hence, in the CPT invariant theories, one has to add the states with the opposite helicity to the above mentioned ones.

Let us consider some examples. We take $N=1$ and $\lambda=0$. Then one has the following set of states:

$$
\begin{aligned}
& N=1 \quad \lambda=0 \\
& \text { helicity } 0 \frac{1}{2} \stackrel{C P T}{\Longrightarrow} \text { helicity } 0-\frac{1}{2} \\
& \text { \# of states } 11 \quad \text { \# of states } 1
\end{aligned}
$$

Hence, the complete $N=1$ multiplet is

$$
\begin{array}{clccc}
N=1 & \text { helicity } & -1 / 2 & 0 & 1 / 2 \\
& \text { \# of states } & 1 & 2 & 1
\end{array}
$$

which contains one complex scalar and one spinor with two helicity states. The number of bosonic degrees of freedom equals to the number of fermionic ones as expected.

This is an example of the so-called self-conjugated multiplet. There are also the self-conjugated multiplets with $N>1$ corresponding to the extended supersymmetry. Two particular examples are the $N=4$ super Yang-Mills multiplet and the $N=8$ supergravity multiplet which contain the states with $\operatorname{spin}=1$ and $\operatorname{spin}=2$, respectively 


$$
\begin{array}{ccccccccc}
N=4 & \text { SUSY YM } & \lambda=-1 \\
\text { helicity } & -1 & -1 / 2 & 0 & 1 / 2 & 1 \\
\text { \# of states } & 1 & 4 & 6 & 4 & 1 \\
\text { N } & \text { SUGRA } & \lambda= & -2 \\
-2 & -3 / 2 & -1 & -1 / 2 & 0 & 1 / 2 & 1 & 3 / 2 & 2 \\
1 & 8 & 28 & 56 & 70 & 56 & 28 & 8 & 1
\end{array}
$$

One can see that the multiplets of extended supersymmetry are very rich and contain a vast number of particles.

The constraint on the number of the SUSY generators comes from the requirement of consistency of the corresponding QFT. The number of supersymmetries and the maximal spin of the particle in the multiplet are related by

$$
N \leq 4 S
$$

where $S$ is the maximal spin. Since the theories with the spin greater than 1 are non-renormalizable and the theories with the spin greater than $5 / 2$ have no consistent coupling to gravity, this imposes a constraint on the number of the SUSY generators

$$
\begin{array}{ll}
N \leq 4 & \text { for renormalizable theories (YM) } \\
N \leq 8 & \text { for (super)gravity. }
\end{array}
$$

In what follows, we shall consider the simple supersymmetry, or the $N=1$ supersymmetry, contrary to the extended supersymmetries with $N>1$. In this case, one has the following types of the supermultiplets which are used for the construction of the SUSY generalization of the SM

$$
\begin{aligned}
& (\phi, \psi) \quad\left(\lambda, A_{\mu}\right) \\
& \operatorname{Spin}=0, \operatorname{Spin}=1 / 2 \quad \operatorname{Spin}=1 / 2, \operatorname{Spin}=1 \\
& \text { scalar chiral Majorana vector } \\
& \text { fermion fermion }
\end{aligned}
$$

each of them contains two physical states, one boson and one fermion. They are called chiral and vector multiplets, respectively. To construct the SUSY generalization of the SM one has to put all the particles into these multiplets. For instance, quarks should go into the chiral multiplet and the photon into the vector multiplet.

\section{Superspace and supermultiplets}

An elegant formulation of the supersymmetry transformations and invariants can be achieved in the framework of the superspace formalism [4]. The superspace differs from the ordinary Euclidean (Minkowski) space by adding two new coordinates, $\theta_{\alpha}$ and $\bar{\theta}_{\dot{\alpha}}$, which are Grassmannian, i. e. anticommuting, variables

$$
\begin{gathered}
\left\{\theta_{\alpha}, \theta_{\beta}\right\}=0, \quad\left\{\bar{\theta}_{\dot{\alpha}}, \bar{\theta}_{\dot{\beta}}\right\}=0, \quad \theta_{\alpha}^{2}=0, \quad \bar{\theta}_{\dot{\alpha}}^{2}=0, \\
\alpha, \beta, \dot{\alpha}, \dot{\beta}=1,2 .
\end{gathered}
$$


Thus, we go from the space to the superspace

$$
\begin{array}{cc}
\text { Space } & \text { Superspace } \\
x_{\mu} & x_{\mu}, \theta_{\alpha}, \bar{\theta}_{\dot{\alpha}}
\end{array}
$$

A SUSY group element can be constructed in the superspace in the same way as the ordinary translation in the usual space

$$
G(x, \theta, \bar{\theta})=e^{i\left(-x^{\mu} P_{\mu}+\theta Q+\bar{\theta} \bar{Q}\right)}
$$

It leads to a supertranslation in the superspace

$$
\begin{aligned}
x_{\mu} & \rightarrow x_{\mu}+i \theta \sigma_{\mu} \bar{\varepsilon}-i \varepsilon \sigma_{\mu} \bar{\theta}, \\
\theta & \rightarrow \theta+\varepsilon \\
\bar{\theta} & \rightarrow \bar{\theta}+\bar{\varepsilon}
\end{aligned}
$$

where $\varepsilon$ and $\bar{\varepsilon}$ are the Grassmannian transformation parameters. From Eqn. (4.2) one can easily obtain the representation for the supercharges (2.4) acting on the superspace

$$
Q_{\alpha}=\frac{\partial}{\partial \theta_{\alpha}}-i \sigma_{\alpha \dot{\alpha}}^{\mu} \bar{\theta}^{\dot{\alpha}} \partial_{\mu}, \quad \bar{Q}_{\dot{\alpha}}=-\frac{\partial}{\partial \bar{\theta}_{\dot{\alpha}}}+i \theta_{\alpha} \sigma_{\alpha \dot{\alpha}}^{\mu} \partial_{\mu} .
$$

To define the fields on a superspace, consider representations of the Super-Poincare group (2.4) [3]. The simplest one is a scalar superfield $F(x, \theta, \bar{\theta})$ which is SUSY invariant. Its Taylor expansion in $\theta$ and $\bar{\theta}$ has only several terms due to the nilpotent character of Grassmannian parameters. However, this superfield is a reducible representation of SUSY. To get an irreducible one, we define a chiral superfield which obeys the equation

$$
\bar{D}_{\dot{\alpha}} \Phi=0, \quad \text { where } \bar{D}_{\dot{\alpha}}=-\frac{\partial}{\partial \bar{\theta}^{\dot{\alpha}}}-i \theta^{\alpha} \sigma_{\alpha \dot{\alpha}}^{\mu} \partial_{\mu}
$$

is a superspace covariant derivative.

For the chiral superfield Grassmannian Taylor expansion looks like $(y=x+i \theta \sigma \bar{\theta})$

$$
\begin{aligned}
\Phi(y, \theta) & =A(y)+\sqrt{2} \theta \psi(y)+\theta \theta F(y)= \\
& =A(x)+i \theta \sigma^{\mu} \bar{\theta} \partial_{\mu} A(x)+\frac{1}{4} \theta \theta \bar{\theta} \bar{\theta} \square A(x) \\
& +\sqrt{2} \theta \psi(x)-\frac{i}{\sqrt{2}} \theta \theta \partial_{\mu} \psi(x) \sigma^{\mu} \bar{\theta}+\theta \theta F(x) .
\end{aligned}
$$

The coefficients are the ordinary functions of $x$ being the usual fields. They are called the components of the superfield. In Eqn. (4.5) one has 2 bosonic (the complex scalar field $A$ ) and 2 fermionic (the Weyl spinor field $\psi$ ) degrees of freedom. The component fields $A$ and $\psi$ are called the $s u$ perpartners. The field $F$ is an auxiliary field, it has the "wrong" dimension and has no physical meaning. It is needed to close the algebra (2.4). One can get rid of the auxiliary fields with the help of equations of motion. 
Thus, the superfield contains an equal number of the bosonic and fermionic degrees of freedom. Under the SUSY transformation they convert one into another

$$
\begin{aligned}
\delta_{\varepsilon} A & =\sqrt{2} \varepsilon \psi, \\
\delta_{\varepsilon} \psi & =i \sqrt{2} \sigma^{\mu} \bar{\varepsilon} \partial_{\mu} A+\sqrt{2} \varepsilon F, \\
\delta_{\varepsilon} F & =i \sqrt{2} \bar{\varepsilon} \sigma^{\mu} \partial_{\mu} \psi .
\end{aligned}
$$

Notice that the variation of the $F$-component is a total derivative, i. e. it vanishes when integrated over the space-time.

One can also construct an antichiral superfield $\Phi^{+}$obeying the equation

$$
D_{\alpha} \Phi^{+}=0, \text { with } D_{\alpha}=\frac{\partial}{\partial \theta^{\alpha}}+i \sigma_{\alpha \dot{\alpha}}^{\mu} \bar{\theta}^{\dot{\alpha}} \partial_{\mu} .
$$

The product of chiral (antichiral) superfields $\Phi^{2}, \Phi^{3}$, etc is also a chiral (antichiral) superfield, while the product of chiral and antichiral ones $\Phi^{+} \Phi$ is a general superfield.

For any arbitrary function of chiral superfields one has

$$
\begin{aligned}
\mathscr{W}\left(\Phi_{i}\right) & =\mathscr{W}\left(A_{i}+\sqrt{2} \theta \psi_{i}+\theta \theta F\right) \\
& =\mathscr{W}\left(A_{i}\right)+\frac{\partial \mathscr{W}}{\partial A_{i}} \sqrt{2} \theta \psi_{i}+\theta \theta\left(\frac{\partial \mathscr{W}}{\partial A_{i}} F_{i}-\frac{1}{2} \frac{\partial^{2} \mathscr{W}}{\partial A_{i} \partial A_{j}} \psi_{i} \psi_{j}\right) .
\end{aligned}
$$

The $\mathscr{W}$ is usually referred to as a superpotential which replaces the usual potential for the scalar fields.

To construct the gauge invariant interactions, one needs a real vector superfield $V=V^{+}$. It is not chiral but rather a general superfield with the following Grassmannian expansion:

$$
\begin{aligned}
V(x, \theta, \bar{\theta}) & =C(x)+i \theta \chi(x)-i \bar{\theta} \bar{\chi}(x)+\frac{i}{2} \theta \theta[M(x)+i N(x)] \\
& -\frac{i}{2} \bar{\theta} \bar{\theta}[M(x)-i N(x)]-\theta \sigma^{\mu} \bar{\theta} v_{\mu}(x)+i \theta \theta \bar{\theta}\left[\lambda(x)+\frac{i}{2} \bar{\sigma}^{\mu} \partial_{\mu} \chi(x)\right] \\
& -i \bar{\theta} \bar{\theta} \theta\left[\lambda+\frac{i}{2} \sigma^{\mu} \partial_{\mu} \bar{\chi}(x)\right]+\frac{1}{2} \theta \theta \bar{\theta} \bar{\theta}\left[D(x)+\frac{1}{2} \square C(x)\right] .
\end{aligned}
$$

The physical degrees of freedom corresponding to the real vector superfield $V$ are the vector gauge field $v_{\mu}$ and the Majorana spinor field $\lambda$. All other components are unphysical and can be eliminated. Indeed, under the Abelian (super)gauge transformation the superfield $V$ is transformed as

$$
V \rightarrow V+\Phi+\Phi^{+}
$$

where $\Phi$ and $\Phi^{+}$are some chiral superfields. In components it looks like

$$
\begin{aligned}
C & \rightarrow C+A+A^{*}, \\
\chi & \rightarrow \chi-i \sqrt{2} \psi, \\
M+i N & \rightarrow M+i N-2 i F, \\
v_{\mu} & \rightarrow v_{\mu}-i \partial_{\mu}\left(A-A^{*}\right), \\
\lambda & \rightarrow \lambda, \\
D & \rightarrow D
\end{aligned}
$$


and corresponds to ordinary gauge transformations for physical components. According to Eqn.(4.9), one can choose a gauge (the Wess-Zumino gauge) where $C=\chi=M=N=0$, leaving one with only physical degrees of freedom except for the auxiliary field $D$. In this gauge

$$
\begin{aligned}
V & =-\theta \sigma^{\mu} \bar{\theta} v_{\mu}(x)+i \theta \theta \bar{\theta} \bar{\lambda}(x)-i \bar{\theta} \bar{\theta} \theta \lambda(x)+\frac{1}{2} \theta \theta \bar{\theta} \bar{\theta} D(x), \\
V^{2} & =-\frac{1}{2} \theta \theta \bar{\theta} \bar{\theta} v_{\mu}(x) v^{\mu}(x), \\
V^{3} & =0, \text { etc. }
\end{aligned}
$$

One can define also a field strength tensor (as the analog of $F_{\mu \nu}$ in the gauge theories)

$$
W_{\alpha}=-\frac{1}{4} \bar{D}^{2} e^{V} D_{\alpha} e^{-V}, \quad \bar{W}_{\dot{\alpha}}=-\frac{1}{4} D^{2} e^{V} \bar{D}_{\alpha} e^{-V},
$$

Here $D_{\alpha}$ and $\bar{D}_{\dot{\alpha}}$ are the supercovariant derivatives

$$
D_{\alpha}=\frac{\partial}{\partial \theta^{\alpha}}+i \sigma_{\alpha \dot{\alpha}}^{\mu} \bar{\theta}^{\dot{\alpha}} \partial_{\mu}, \quad \bar{D}_{\dot{\alpha}}=-\frac{\partial}{\partial \bar{\theta}^{\dot{\alpha}}}-i \theta^{\alpha} \sigma_{\alpha \dot{\alpha}}^{\mu} \partial_{\mu} .
$$

The field strength tensor in the chosen Wess-Zumino gauge is a polynomial over the component fields:

$$
W_{\alpha}=T^{a}\left(-i \lambda_{\alpha}^{a}+\theta_{\alpha} D^{a}-\frac{i}{2}\left(\sigma^{\mu} \bar{\sigma}^{v} \theta\right)_{\alpha} F_{\mu \nu}^{a}+\theta^{2}\left(\sigma^{\mu} D_{\mu} \bar{\lambda}^{a}\right)_{\alpha}\right),
$$

where

$$
F_{\mu \nu}^{a}=\partial_{\mu} v_{v}^{a}-\partial_{\nu} v_{\mu}^{a}+f^{a b c} v_{\mu}^{b} v_{v}^{c}, \quad D_{\mu} \bar{\lambda}^{a}=\partial \bar{\lambda}^{a}+f^{a b c} v_{\mu}^{b} \bar{\lambda}^{c} .
$$

In the Abelian case Eqs. (4.11) are simplified and take the form

$$
W_{\alpha}=-\frac{1}{4} \bar{D}^{2} D_{\alpha} V, \quad \bar{W}_{\dot{\alpha}}=-\frac{1}{4} D^{2} \bar{D}_{\alpha} V .
$$

\section{Construction of SUSY Lagrangians}

Let us start with the Lagrangian which has no local gauge invariance. In the superfield notation the SUSY invariant Lagrangians are the polynomials of the superfields. In the same way, as the ordinary action is the integral over the space-time of the Lagrangian density, in the supersymmetric case the action is the integral over the superspace. The space-time Lagrangian density is $[3,4]$

$$
\mathscr{L}=\int d^{2} \theta d^{2} \bar{\theta} \Phi_{i}^{+} \Phi_{i}+\int d^{2} \theta\left[\lambda_{i} \Phi_{i}+\frac{1}{2} m_{i j} \Phi_{i} \Phi_{j}+\frac{1}{3} y_{i j k} \Phi_{i} \Phi_{j} \Phi_{k}\right]+\text { h.c. }
$$

where the first part is the kinetic term and the second one is the superpotential $\mathscr{W}$. We use here the integration over the superspace according to the rules of the Grassmannian integration [8]

$$
\int d \theta_{\alpha}=0, \quad \int \theta_{\alpha} d \theta_{\beta}=\delta_{\alpha \beta}
$$

Performing the explicit integration over the Grassmannian parameters, we get from Eqn. (5.1)

$$
\begin{aligned}
\mathscr{L} & =i \partial_{\mu} \bar{\psi}_{i} \bar{\sigma}^{\mu} \psi_{i}+A_{i}^{*} \square A_{i}+F_{i}^{*} F_{i} \\
& +\left[\lambda_{i} F_{i}+m_{i j}\left(A_{i} F_{j}-\frac{1}{2} \psi_{i} \psi_{j}\right)+y_{i j k}\left(A_{i} A_{j} F_{k}-\psi_{i} \psi_{j} A_{k}\right)+\text { h.c. }\right] .
\end{aligned}
$$


The last two terms are the interaction ones. To obtain the familiar form of the Lagrangian, we have to solve the constraints

$$
\begin{aligned}
& \frac{\partial \mathscr{L}}{\partial F_{k}^{*}}=F_{k}+\lambda_{k}^{*}+m_{i k}^{*} A_{i}^{*}+y_{i j k}^{*} A_{i}^{*} A_{j}^{*}=0, \\
& \frac{\partial \mathscr{L}}{\partial F_{k}}=F_{k}^{*}+\lambda_{k}+m_{i k} A_{i}+y_{i j k} A_{i} A_{j}=0 .
\end{aligned}
$$

Expressing the auxiliary fields $F$ and $F^{*}$ from these equations, one finally gets

$$
\begin{aligned}
\mathscr{L} & =i \partial_{\mu} \bar{\psi}_{i} \bar{\sigma}^{\mu} \psi_{i}+A_{i}^{*} \square A_{i}-\frac{1}{2} m_{i j} \psi_{i} \psi_{j}-\frac{1}{2} m_{i j}^{*} \bar{\psi}_{i} \bar{\psi}_{j} \\
& -y_{i j k} \psi_{i} \psi_{j} A_{k}-y_{i j k}^{*} \bar{\psi}_{i} \bar{\psi}_{j} A_{k}^{*}-V\left(A_{i}, A_{j}\right),
\end{aligned}
$$

where the scalar potential $V=F_{k}^{*} F_{k}$. We will return to the discussion of the form of the scalar potential in the SUSY theories later.

Consider now the gauge invariant SUSY Lagrangians. They should contain the gauge invariant interaction of the matter fields with the gauge ones and the kinetic term and the self-interaction of the gauge fields.

Let us start with the gauge field kinetic terms. In the Wess-Zumino gauge one has

$$
\left.W^{\alpha} W_{\alpha}\right|_{\theta \theta}=-2 i \lambda \sigma^{\mu} D_{\mu} \bar{\lambda}-\frac{1}{2} F_{\mu v} F^{\mu v}+\frac{1}{2} D^{2}+\frac{i}{4} F^{\mu v} F^{\rho \sigma} \varepsilon_{\mu v \rho \sigma},
$$

where $D_{\mu} \bar{\lambda}=\partial_{\mu}+i g\left[v_{\mu}, \bar{\lambda}\right]$ is the usual covariant derivative and the last, the so-called topological $\theta$-term, is the total derivative. The gauge invariant Lagrangian now has the familiar form

$$
\begin{aligned}
\mathscr{L} & =\frac{1}{4} \int d^{2} \theta W^{\alpha} W_{\alpha}+\frac{1}{4} \int d^{2} \bar{\theta} \bar{W}^{\dot{\alpha}} \bar{W}_{\dot{\alpha}} \\
& =\frac{1}{2} D^{2}-\frac{1}{4} F_{\mu v} F^{\mu v}-i \lambda \sigma^{\mu} D_{\mu} \bar{\lambda} .
\end{aligned}
$$

To obtain the gauge-invariant interaction with the matter chiral superfields, one has to modify the kinetic term by inserting the bridge operator

$$
\Phi_{i}^{+} \Phi_{i} \Longrightarrow \Phi_{i}^{+} e^{g V} \Phi_{i} .
$$

The complete SUSY and gauge invariant Lagrangian then looks like

$$
\begin{aligned}
\mathscr{L}_{\text {SUSY YM }} & =\frac{1}{4} \int d^{2} \theta \operatorname{Tr}\left(W^{\alpha} W_{\alpha}\right)+\frac{1}{4} \int d^{2} \bar{\theta} \operatorname{Tr}\left(\bar{W}^{\alpha} \bar{W}_{\alpha}\right) \\
& +\int d^{2} \theta d^{2} \bar{\theta} \bar{\Phi}_{i a}\left(e^{g V}\right)_{b}^{a} \Phi_{i}^{b}+\int d^{2} \theta \mathscr{W}\left(\Phi_{i}\right)+\int d^{2} \bar{\theta} \overline{\mathscr{W}}\left(\bar{\Phi}_{i}\right),
\end{aligned}
$$

where $\mathscr{W}$ is the superpotential, which should be invariant under the group of symmetry of the particular model. In terms of thecomponent fields the above Lagrangian takes the form

$$
\begin{aligned}
\mathscr{L}_{\text {SUSY YM }} & =-\frac{1}{4} F_{\mu \nu}^{a} F^{a \mu \nu}-i \lambda^{a} \sigma^{\mu} D_{\mu} \bar{\lambda}^{a}+\frac{1}{2} D^{a} D^{a} \\
& +\left(\partial_{\mu} A_{i}-i g v_{\mu}^{a} T^{a} A_{i}\right)^{\dagger}\left(\partial_{\mu} A_{i}-i g v_{\mu}^{a} T^{a} A_{i}\right)-i \bar{\psi}_{i} \bar{\sigma}^{\mu}\left(\partial_{\mu} \psi_{i}-i g v_{\mu}^{a} T^{a} \psi_{i}\right) \\
& -D^{a} A_{i}^{\dagger} T^{a} A_{i}-i \sqrt{2} A_{i}^{\dagger} T^{a} \lambda^{a} \psi_{i}+i \sqrt{2} \bar{\psi}_{i} T^{a} A_{i} \bar{\lambda}^{a}+F_{i}^{\dagger} F_{i} \\
& +\frac{\partial \mathscr{W}}{\partial A_{i}} F_{i}+\frac{\partial \overline{\mathscr{W}}}{\partial A_{i}^{\dagger}} F_{i}^{\dagger}-\frac{1}{2} \frac{\partial^{2} \mathscr{W}}{\partial A_{i} \partial A_{j}} \psi_{i} \psi_{j}-\frac{1}{2} \frac{\partial^{2} \overline{\mathscr{W}}_{\partial A_{i}^{\dagger} \partial A_{j}^{\dagger}} \bar{\psi}_{i} \bar{\psi}_{j} .}{} \text {. }
\end{aligned}
$$

Integrating out the auxiliary fields $D^{a}$ and $F_{i}$, one reproduces the usual Lagrangian. 


\section{The scalar potential}

Contrary to the SM, where the scalar potential is arbitrary and is defined only by the requirement of the gauge invariance, in the supersymmetric theories it is completely defined by the superpotential. It consists of the contributions from the $D$-terms and $F$-terms. The kinetic energy of the gauge fields (recall Eqn. (5.6) yields the $\frac{1}{2} D^{a} D^{a}$ term, and the matter-gauge interaction (recall Eqn. (5.9) yields the $g D^{a} T_{i j}^{a} A_{i}^{*} A_{j}$ one. Together they give

$$
\mathscr{L}_{D}=\frac{1}{2} D^{a} D^{a}+g D^{a} T_{i j}^{a} A_{i}^{*} A_{j} .
$$

The equation of motion reads

$$
D^{a}=-g T_{i j}^{a} A_{i}^{*} A_{j} .
$$

Substituting it back into Eqn. (6.1) yields the $D$-term part of the potential

$$
\mathscr{L}_{D}=-\frac{1}{2} D^{a} D^{a} \Longrightarrow V_{D}=\frac{1}{2} D^{a} D^{a},
$$

where $D$ is given by Eqn. (6.2).

The $F$-term contribution can be derived from the matter field self-interaction (5.2). For a general type superpotential $\mathscr{W}$ one has

$$
\mathscr{L}_{F}=F_{i}^{*} F_{i}+\left(\frac{\partial W}{\partial A_{i}} F_{i}+\text { h.c. }\right) .
$$

Using the equations of motion for the auxiliary field $F_{i}$

$$
F_{i}^{*}=-\frac{\partial W}{\partial A_{i}}
$$

yields

$$
\mathscr{L}_{F}=-F_{i}^{*} F_{i} \Longrightarrow V_{F}=F_{i}^{*} F_{i},
$$

where $F$ is given by Eqn. (6.5). The full scalar potential is the sum of the two contributions

$$
V=V_{D}+V_{F} .
$$

Thus, the form of the Lagrangian is practically fixed by the symmetry requirements. The only freedom is the field content, the value of the gauge coupling $g$, Yukawa couplings $y_{i j k}$ and the masses. Because of the renormalizability constraint $V \leq A^{4}$ the superpotential should be limited by $\mathscr{W} \leq \Phi^{3}$ as in Eqn. (5.1). All members of the supermultiplet have the same masses, i. e. the bosons and the fermions are degenerate in masses. This property of the SUSY theories contradicts to the phenomenology and requires supersymmetry breaking.

\section{Spontaneous breaking of SUSY}

Since supersymmetric algebra leads to mass degeneracy in a supermultiplet, it should be broken to explain the absence of superpartners at modern energies. There are several ways of supersymmetry breaking. It can be broken either explicitly or spontaneously. Performing SUSY 
breaking one has to be careful not to spoil the cancellation of quadratic divergencies which allows one to solve the hierarchy problem. This is achieved by spontaneous breaking of SUSY.

Apart from non-supersymmetric theories in SUSY models the energy is always nonnegative definite. Indeed, according to quantum mechanics

$$
E=<0|H| 0>
$$

and due to SUSY algebra eq.(2.4)

$$
\left\{Q_{\alpha}, \bar{Q}_{\dot{\beta}}\right\}=2\left(\sigma^{\mu}\right)_{\alpha \dot{\beta}} P_{\mu},
$$

taking into account that $\operatorname{tr}\left(\sigma^{\mu} P_{\mu}\right)=2 P_{0}$, one gets

$$
E=\frac{1}{4} \sum_{\alpha=1,2}<0\left|\left\{Q_{\alpha}, \bar{Q}_{\alpha}\right\}\right| 0>=\frac{1}{4} \sum_{\alpha}\left|Q_{\alpha}\right| 0>\left.\right|^{2} \geq 0 .
$$

Hence

$$
E=<0|H| 0>\neq 0 \text { if and only if } Q_{\alpha} \mid 0>\neq 0 .
$$

Therefore, supersymmetry is spontaneously broken, i.e. vacuum is not invariant $\left(Q_{\alpha} \mid 0>\neq 0\right)$, if and only if the minimum of the potential is positive (i.e. $E>0)$.

The situation is illustrated in Fig.1. The SUSY ground state has $E=0$, while a non-SUSY one has $E>0$. On the right-hand side a non-SUSY potential is shown. It does not appear even in spontaneously broken SUSY theories. However, just this type of the potential is used for spontaneous breaking of the gauge invariance via the Higgs mechanism. This property has crucial consequences for the spontaneous breaking of the gauge invariance. Indeed, as will be seen later, in the MSSM spontaneous breaking of $S U(2)$ invariance takes place only after SUSY is broken.
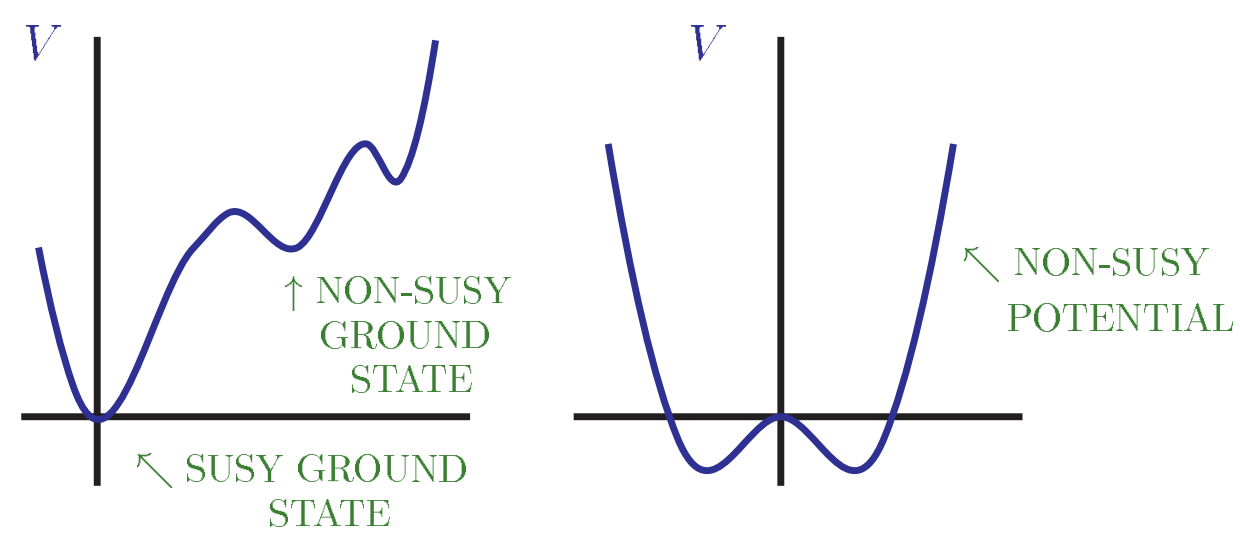

Figure 1: Scalar potential in supersymmetric and non-supersymmetric theories

Spontaneous breaking of supersymmetry is achieved in the same way as the electroweak symmetry breaking. One introduces the field whose vacuum expectation value is nonzero and breaks the symmetry. However, due to a special character of SUSY, this should be a superfield whose auxiliary $F$ and $D$ components acquire nonzero v.e.v.'s. Thus, among possible spontaneous SUSY breaking mechanisms one distinguishes the $F$ and $D$ ones. 
i) Fayet-Iliopoulos (D-term) mechanism [9].

In this case the, the linear $D$-term is added to the Lagrangian

$$
\Delta \mathscr{L}=\left.\xi V\right|_{\theta \theta \bar{\theta} \bar{\theta}}=\xi \int d^{4} \theta V .
$$

It is gauge and SUSY invariant by itself; however, it may lead to spontaneous breaking of both of them depending on the value of $\xi$. We show in Fig.2a the sample spectrum for two chiral matter multiplets. The drawback of this mechanism is the necessity of $U(1)$ gauge invariance. It can be

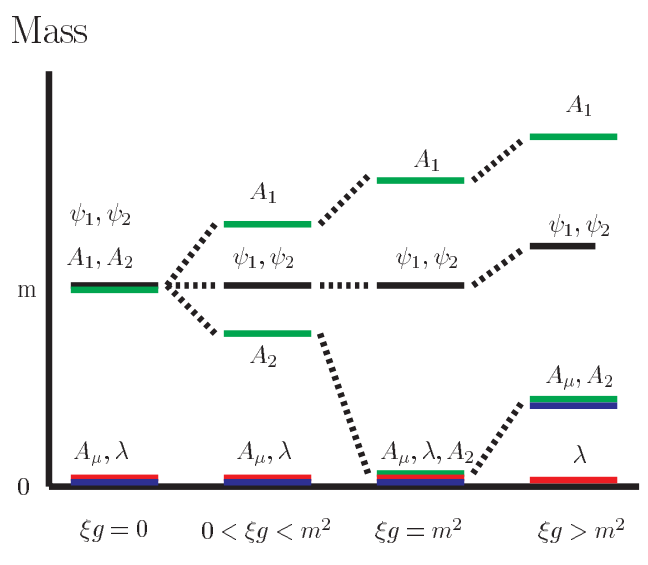

a)

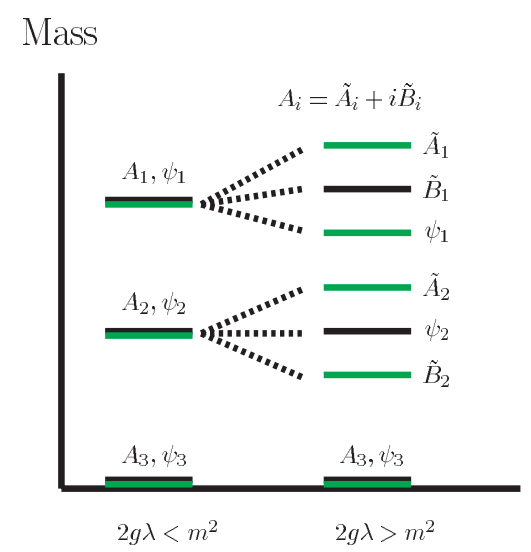

b)

Figure 2: Spectrum of spontaneously broken SUSY theories

used in SUSY generalizations of the SM but not in GUTs.

The mass spectrum also causes some troubles since the following sum rule is always valid

$$
\sum_{\text {boson states }} m_{i}^{2}=\sum_{\text {fermion states }} m_{i}^{2}
$$

which is bad for phenomenology.

ii) O'Raifeartaigh ( $F$-term) mechanism [10].

In this case, several chiral fields are needed and the superpotential should be chosen in a way that trivial zero v.e.v.s for the auxiliary $F$-fields be absent. For instance, choosing the superpotential to be

$$
\mathscr{W}(\Phi)=\lambda \Phi_{3}+m \Phi_{1} \Phi_{2}+g \Phi_{3} \Phi_{1}^{2}
$$

one gets the equations for the auxiliary fields

$$
\begin{aligned}
& F_{1}^{*}=m A_{2}+2 g A_{1} A_{3}, \\
& F_{2}^{*}=m A_{1}, \\
& F_{3}^{*}=\lambda+g A_{1}^{2},
\end{aligned}
$$

which have no solutions with $\left.<F_{i}\right\rangle=0$ and SUSY is spontaneously broken. The sample spectrum is shown in Fig.2b.

The drawbacks of this mechanism is a lot of arbitrariness in the choice of potential. The sum rule (7.2) is also valid here. 
Unfortunately, none of these mechanisms explicitly works in SUSY generalizations of the SM. None of the fields of the SM can develop nonzero v.e.v.s for their $F$ or $D$ components without breaking $S U(3)$ or $U(1)$ gauge invariance since they are not singlets with respect to these groups. This requires the presence of extra sources of spontaneous SUSY breaking, which we consider below. They are based, however, on the same $F$ and $D$ mechanisms.

\section{PART II: Supersymmetric Standard Model}

\section{Motivation for SUSY in particle physics}

\subsection{Unification with gravity}

Let us consider the infinitesimal SUSY transformations $\delta_{\varepsilon}=\varepsilon^{\alpha} Q_{\alpha}, \bar{\delta}_{\bar{\varepsilon}}=\bar{Q}_{\dot{\alpha}} \bar{\varepsilon}^{\dot{\alpha}}$. Then according to SUSY algebra one gets

$$
\left\{\delta_{\varepsilon}, \bar{\delta}_{\bar{\varepsilon}}\right\}=2\left(\varepsilon \sigma^{\mu} \bar{\varepsilon}\right) P_{\mu},
$$

where $\varepsilon, \bar{\varepsilon}$ are transformation parameters. Choosing $\varepsilon$ to be local, i. e. the function of the spacetime point $\varepsilon=\varepsilon(x)$, one finds from eqn. (1.1) that the anticommutator of two SUSY transformations is a local coordinate translation, and the theory which is invariant under the local coordinate transformation is the General Relativity. Thus, making SUSY local, one naturally obtains the General Relativity, or the theory of gravity, or supergravity [2].

\subsection{Unification of gauge couplings}

According to the Grand Unification hypothesis, the gauge symmetry increases with the energy [11]. All known interactions are different branches of the unique interaction associated with a simple gauge group. The unification (or splitting) occurs at the high energy. To reach this goal one has to consider how the couplings change with the energy. It is described by renormalization group equations. In the SM the strong and weak couplings associated with the non-Abelian gauge groups decrease with the energy, while the electromagnetic one associated with the Abelian group on the contrary increases. Thus, it is possible that at some energy scale they are equal.

After the precise measurement of the $S U(3) \times S U(2) \times U(1)$ coupling constants, it has become possible to check the unification numerically. The three coupling constants to be compared are

$$
\begin{aligned}
& \alpha_{1}=(5 / 3) g^{2} /(4 \pi)=5 \alpha /\left(3 \cos ^{2} \theta_{W}\right), \\
& \alpha_{2}=g^{2} /(4 \pi)=\alpha / \sin ^{2} \theta_{W}, \\
& \alpha_{3}=g_{s}^{2} /(4 \pi)
\end{aligned}
$$

where $g^{\prime}, g$ and $g_{s}$ are the usual $U(1), S U(2)$ and $S U(3)$ couplings and $\alpha$ is the fine structure constant. The factor of $5 / 3$ in $\alpha_{1}$ has been included for proper normalization of the generators.

In the modified minimal subtraction $(\overline{M S})$ scheme, the world averaged values of the couplings at the $Z^{0}$ energy are obtained from the fit to the LEP and Tevatron data [12]:

$$
\begin{aligned}
& \alpha_{1}\left(M_{Z}\right)=0.017 \\
& \alpha_{2}\left(M_{Z}\right)=0.034 \\
& \alpha_{3}\left(M_{Z}\right)=0.118 \pm 0.003 .
\end{aligned}
$$


Assuming that the SM is valid up to the unification scale, one can then use the known RG equations for the three couplings. In the leading one has:

$$
\frac{1}{\tilde{\alpha}_{i}\left(Q^{2}\right)}=\frac{1}{\tilde{\alpha}_{i}\left(\mu^{2}\right)}-b_{i} \log \left(\frac{Q^{2}}{\mu^{2}}\right)
$$

\section{Unification of the Coupling Constants in the SM and the minimal MSSM}
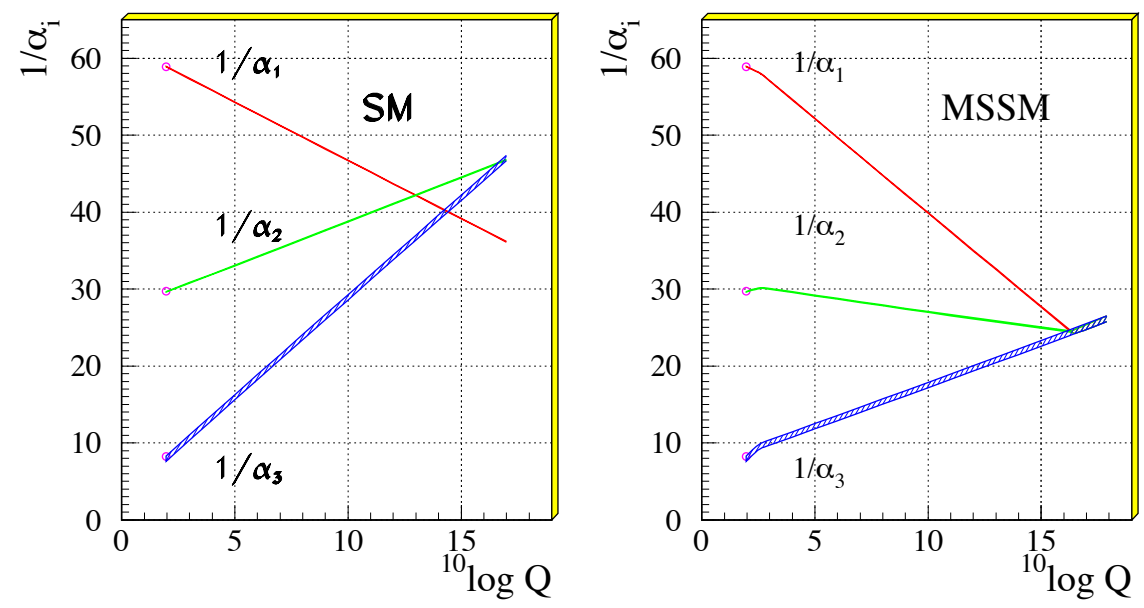

Figure 3: The evolution of the inverse of the three coupling constants in the Standard Model (left) and in the supersymmetric extension of the SM (MSSM) (right).

The result is demonstrated in Fig. 3 [13] showing the evolution of the inverse of the couplings as a function of the logarithm of energy. In this presentation, the evolution becomes a straight line in the first order. The second order corrections are small and do not cause any visible deviation from the straight line. Fig. 3 clearly demonstrates that within the SM the coupling constant unification at a single point is impossible. It is excluded by more than 8 standard deviations. This result means that the unification can only be obtained if the new physics enters between the electroweak and the Planck scales.

In the SUSY case, the slopes of the RG evolution curves are modified. The SUSY particles are assumed to contribute effectively to the running of the coupling constants only for the energies above the typical SUSY mass scale. It turns out that within the SUSY model the perfect unification can be obtained as it is shown in Fig. 3. What is remarkable the SUSY scale happened to be in the $\mathrm{TeV}$ range offering hopes for the experimental observation of SUSY.

\subsection{Solution to the hierarchy problem}

The appearance of two different scales $V \gg v$ in the GUT theory, namely, $M_{G U T}$ and $M_{W}$, leads to a very serious problem which is called the hierarchy problem. There are two aspects of this problem. 
The first one is the very existence of the hierarchy. To get the desired spontaneous symmetry breaking pattern, one needs

$$
\begin{array}{ll}
m_{H} & \sim v \sim 10^{2} \mathrm{GeV} \\
m_{\Sigma} & \sim V \sim 10^{16} \mathrm{GeV}
\end{array} \quad \frac{m_{H}}{m_{\Sigma}} \sim 10^{-14} \ll 1,
$$

where $H$ and $\Sigma$ are the Higgs fields responsible for the spontaneous breaking of the $S U(2)$ and GUT group, respectively. The question arises of how to get so small number in a natural way.

The second aspect of the hierarchy problem is connected with the preservation of the given hierarchy. Even if we choose the hierarchy like in Eqn. (1.5) the radiative corrections will destroy it! The very accurate cancelation with a precision $\sim 10^{-14}$ needs a fine-tuning of the coupling constants.

The only known way of achieving this kind of cancelation of quadratic terms (also known as the cancelation of the quadratic divergencies) is supersymmetry. Moreover, SUSY automatically cancels the quadratic corrections in all orders of the perturbation theory. This is due to the contributions of superpartners of ordinary particles. The contribution from boson loops cancels those from the fermion ones because of an additional factor (-1) coming from the Fermi statistics, as shown in Fig. 4.

One can see here two types of contribution. The first line is the contribution of the heavy Higgs boson and its superpartner (higgsino). The strength of the interaction is given by the Yukawa coupling constant $\lambda$. The second line represents the gauge interaction proportional to the gauge coupling constant $g$ with the contribution from the heavy gauge boson and its heavy superpartner (gaugino).

In both cases the cancelation of the quadratic terms takes place. This cancelation is true up to the SUSY breaking scale, $M_{S U S Y}$, which should not be very large ( $\leq 1 \mathrm{TeV}$ ) to make the fine-tuning natural. Indeed, let us take the Higgs boson mass. Requiring for consistency of the perturbation theory that the radiative corrections to the Higgs boson mass do not exceed the mass itself gives

$$
\delta M_{h}^{2} \sim g^{2} M_{S U S Y}^{2} \sim M_{h}^{2}
$$
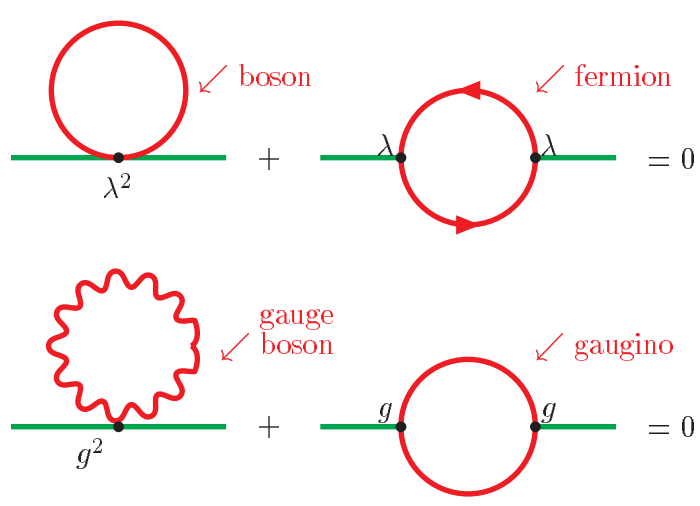

Figure 4: Cancellation of the quadratic terms (divergencies). 
So, if $M_{h} \sim 10^{2} \mathrm{GeV}$ and $g \sim 10^{-1}$, one needs $M_{S U S Y} \sim 10^{3} \mathrm{GeV}$ in order that the relation (1.6) is valid. Thus, we again get the same rough estimate of $M_{S U S Y} \sim 1 \mathrm{TeV}$ as from the gauge coupling unification above.

That is why it is usually said that supersymmetry solves the hierarchy problem. We show below how SUSY can also explain the origin of the hierarchy.

\subsection{Explanation of the EW symmetry breaking}

To break the Electroweak symmetry we use the Brout-Englert-Higgs mechanism os spontaneous symmetry breaking. However, the form of the scalar field potential is taken ad hoc. Is there any way to explain its appearance? It happens so that SUSY models provide such an explanation. As will be shown below, one originally starts with unbroken potential shown in Fig.5 (left) and then arrives at the famous Mexican hat potential Fig.5 (right) as a result of radiative corrections. Thus, supersymmetry provides the mechanism of radiative EW symmetry breaking in a natural way.

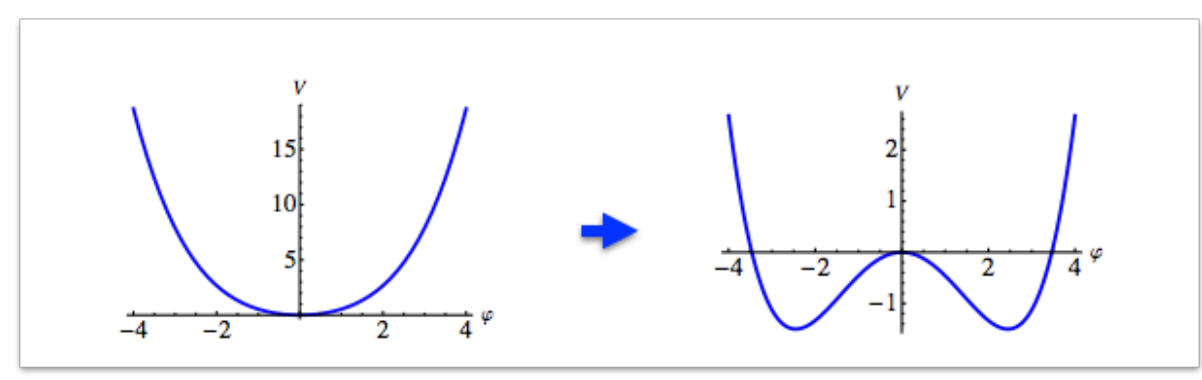

Figure 5: EW symmetry breaking

\subsection{Astrophysics and Cosmology}

The shining matter is not the only one in the Universe. Considerable amount of the energy budget consists of the so-called dark matter. The direct evidence for the presence of the dark matter are flat rotation curves of spiral galaxies [14]. To explain these curves one has to assume the existence of a galactic halo made of non-shining matter which takes part in the gravitational interaction. The other manifestation of existence of the dark matter is the so-called gravitational lensing caused by invisible gravitating matter in the sky [15], which leads to the appearance of circular images of distant stars when the light from them passes through the dark matter.

There are two possible types of the dark matter: the hot one, consisting of light relativistic particles and the cold one, consisting of massive weakly interacting particles (WIMPs) [16]. The hot dark matter might consist of neutrinos, however, this has problems with the galaxy formation. As for the cold dark matter, it has no candidates within the SM. At the same time, SUSY provides an excellent candidate for the cold dark matter, namely, the neutralino, the lightest superparticle [17].

$$
\left|\tilde{\chi}_{1}^{0}\right\rangle=N_{1}\left|B_{0}\right\rangle+N_{2}\left|W_{0}^{3}\right\rangle+N_{3}\left|H_{1}\right\rangle+N_{4}\left|H_{2}\right\rangle \text {. }
$$

It is neutral, heavy, stable and takes part in weak interactions, precisely what is needed for a WIMP. 


\section{SUSY generalization of the Standard Model. The MSSM}

As has been already mentioned, in the SUSY theories the number of the bosonic degrees of freedom equals that of fermionic. At the same time, in the SM one has 28 bosonic and 90 fermionic degrees of freedom (without right-handed neutrino, otherwise 96). So the SM is to a great extent non-supersymmetric. Trying to add some new particles to supersymmetrize the SM, one should take into account the following observations:

- There are no fermions with quantum numbers of the gauge bosons;

- Higgs fields have nonzero vacuum expectation values; hence, they cannot be the superpartners of the quarks and leptons, since this would induce a spontaneous violation of the baryon and lepton numbers;

- One needs at least two complex chiral Higgs multiplets in order to give masses to the up and down quarks.

The latter is due to the form of the superpotential and the chirality of the matter superfields. Indeed, the superpotential should be invariant under the $S U(3) \times S U(2) \times U(1)$ gauge group. If one looks at the Yukawa interaction in the Standard Model, one finds that it is indeed $U(1)$ invariant since the sum of hypercharges in each vertex equals zero. For the up quarks this is achieved by taking the conjugated Higgs doublet $\tilde{H}=i \tau_{2} H^{\dagger}$ instead of $H$. However, in SUSY $H$ is the chiral superfield and hence the superpotential which is constructed out of the chiral fields, may contain only $H$ but not $\tilde{H}$ which is the antichiral superfield.

Another reason for the second Higgs doublet is related to chiral anomalies. It is known that the chiral anomalies spoil the gauge invariance and, hence, the renormalizability of the theory. They are canceled in the SM between the quarks and leptons in each generation [18]

$$
\begin{aligned}
\operatorname{Tr} Y^{3}= & 3 \times\left(\frac{1}{27}+\frac{1}{27}-\frac{64}{27}+\frac{8}{27}\right)-1-1+8=0 \\
& \text { color } u_{L} \quad d_{L} \quad u_{R} \quad d_{R} \quad v_{L} e_{L} e_{R}
\end{aligned}
$$

However, if one introduces the chiral Higgs superfield, it contains higgsinos, which are the chiral fermions, and contain the anomalies. To cancel them one has to add the second Higgs doublet with the opposite hypercharge. Therefore, the Higgs sector in the SUSY models is inevitably enlarged, it contains an even number of the Higgs doublets.

Conclusion: In the SUSY models the supersymmetry associates the known bosons with the new fermions and the known fermions with the new bosons.

\subsection{The field content}

Consider the particle content of the Minimal Supersymmetric Standard Model [19, 20, 21]. According to the previous discussion, in the minimal version we double the number of particles (introducing the superpartner to each particle) and add another Higgs doublet (with its superpartner).

Thus, the characteristic feature of any supersymmetric generalization of the SM is the presence of the superpartners (see Fig. 6). If the supersymmetry is exact, the superpartners of the ordinary 


\section{SUPERSYMMETRY}

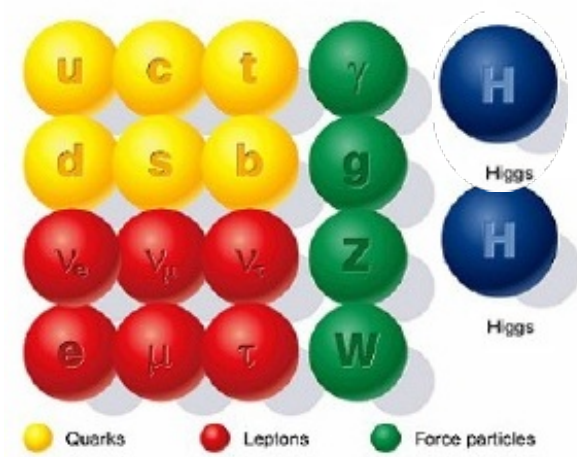

Standard particles

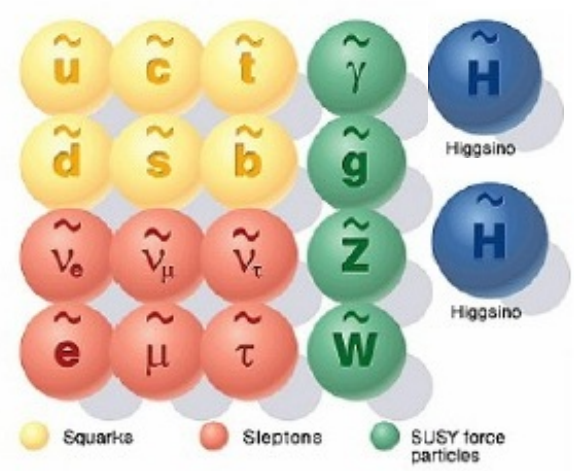

SUSY particles

Figure 6: The world of supersymmetric particles

particles should have the same masses and have to be observed. The absence of them at modern energies is believed to be explained by the fact that they are very heavy, that means that the supersymmetry should be broken. Hence, if the energy of accelerators is high enough, the superpartners will be created.

The particle content of the MSSM then appears as shown in the Table 1. Hereafter, a tilde denotes the superpartner of the ordinary particle.

\begin{tabular}{|c|c|c|c|c|c|c|c|}
\hline \multirow{2}{*}{$\begin{array}{l}\text { Superfield } \\
\text { Gauge }\end{array}$} & \multicolumn{2}{|l|}{ Bosons } & \multicolumn{2}{|c|}{ Fermions } & \multicolumn{3}{|c|}{$S U(3) S U\left(2 U_{Y}(1)\right.$} \\
\hline & & & & & & & \\
\hline $\mathbf{G}^{\mathbf{a}}$ & gluon & $g^{a}$ & gluino & $\tilde{g}^{a}$ & 8 & 0 & 0 \\
\hline $\mathbf{V}^{\mathbf{k}}$ & Weak $W^{k}$ & $\left(W^{ \pm}, Z\right)$ & wino, zino & $\tilde{w}^{k}\left(\tilde{w}^{ \pm}, \tilde{z}\right)$ & 1 & 3 & 0 \\
\hline $\mathbf{V}^{\prime}$ & Hypercharg & $B(\gamma)$ & bino & $\tilde{b}(\tilde{\gamma})$ & 1 & 1 & 0 \\
\hline \multicolumn{8}{|l|}{ Matter } \\
\hline & & $\tilde{L}_{i}=(\tilde{v}, \tilde{e})_{L}$ & & $L_{i}=(v, e)_{L}$ & 1 & 2 & -1 \\
\hline $\begin{array}{l}\mathbf{L}_{\mathbf{i}} \\
\mathbf{F} .\end{array}$ & sleptons & $\tilde{E}_{i}=\tilde{e}_{R}$ & leptons & $E_{i}=e_{R}^{c}$ & 1 & 1 & 2 \\
\hline & & $\tilde{N}_{i}=\tilde{v}_{R}$ & & $N_{i}=v_{R}^{c}$ & 1 & 1 & 0 \\
\hline $\mathbf{Q}_{\mathbf{i}}$ & & $\tilde{Q}_{i}=(\tilde{u}, \tilde{d})_{L}$ & & $Q_{i}=(u, d)_{L}$ & 3 & 2 & $1 / 3$ \\
\hline $\mathbf{U}_{\mathbf{i}}$ & squarks & $\tilde{U}_{i}=\tilde{u}_{R}$ & quarks & $U_{i}=u_{R}^{c}$ & $3^{*}$ & 1 & $-4 / 3$ \\
\hline $\mathbf{D}_{\mathbf{i}}$ & & $\tilde{D}_{i}=\tilde{d}_{R}$ & & $D_{i}=d_{R}^{c}$ & $3^{*}$ & 1 & $2 / 3$ \\
\hline \multicolumn{8}{|l|}{$\overline{\text { Higgs }}$} \\
\hline $\mathbf{H}_{1}$ & Hiqgrec & $H_{1}$ & higa & $\tilde{H}_{1}$ & 1 & 2 & -1 \\
\hline $\mathbf{H}_{2}$ & Higgses & $\mathrm{H}_{2}$ & miggsinos & $\tilde{H}_{2}$ & 1 & 2 & 1 \\
\hline
\end{tabular}

Table 1: Particle content of the MSSM 
The presence of the extra Higgs doublet in the SUSY model is a novel feature of the theory. In the MSSM one has two doublets with the quantum numbers $(1,2,-1)$ and $(1,2,1)$, respectively:

$$
\begin{aligned}
& H_{1}=\left(\begin{array}{c}
H_{1}^{0} \\
H_{1}^{-}
\end{array}\right)=\left(\begin{array}{c}
v_{1}+\frac{S_{1}+i P_{1}}{\sqrt{2}} \\
H_{1}^{-}
\end{array}\right), \\
& H_{2}=\left(\begin{array}{c}
H_{2}^{+} \\
H_{2}^{0}
\end{array}\right)=\left(\begin{array}{c}
H_{2}^{+} \\
v_{2}+\frac{S_{2}+i P_{2}}{\sqrt{2}}
\end{array}\right),
\end{aligned}
$$

where $v_{i}$ are the vacuum expectation values of the neutral components of the Higgs doublets.

Hence, one has $8=4+4=5+3$ degrees of freedom. As in the case of the SM, 3 degrees of freedom can be gauged away, and one is left with 5 physical states compared to 1 in the SM. Thus, in the MSSM, as actually in any two Higgs doublet model, one has five physical Higgs bosons: two $C P$-even neutral Higgs, one $C P$-odd neutral Higgs and two charged ones. We consider the mass eigenstates below.

\subsection{Lagrangian of the MSSM}

Now we can construct the Lagrangian of the MSSM. It consists of two parts; the first part is the SUSY generalization of the Standard Model, while the second one represents the SUSY breaking as mentioned above.

$$
\mathscr{L}_{\text {MSSM }}=\mathscr{L}_{\text {SUSY }}+\mathscr{L}_{\text {Breaking }},
$$

where

$$
\mathscr{L}_{\text {SUSY }}=\mathscr{L}_{\text {Gauge }}+\mathscr{L}_{\text {Yukawa }}
$$

We will not describe the gauge part here, since it is essentially the gauge invariant kinetic terms, but rather concentrate on Yukawa terms. They are given by the superpotential which is nothing else but the usual Yukawa terms of the SM with the fields replaced by the superfields as explained above.

$$
\mathscr{L}_{\text {Yukawa }}=\varepsilon_{i j}\left(y_{a b}^{U} Q_{a}^{j} U_{b}^{c} H_{2}^{i}+y_{a b}^{D} Q_{a}^{j} D_{b}^{c} H_{1}^{i}+y_{a b}^{L} L_{a}^{j} E_{b}^{c} H_{1}^{i}+\mu H_{1}^{i} H_{2}^{j}\right),
$$

where $i, j=1,2$ are the $S U(2)$ and $a, b=1,2,3$ are the generation indices; the $S U(3)$ colour indices are omitted. This part of the Lagrangian almost exactly repeats that of the SM. The only difference is the last term which describes the Higgs mixing. It is absent in the SM since there is only one Higgs field there.

However, one can write down also the different Yukawa terms

$$
\mathscr{L}_{\text {Yukawa }}=\varepsilon_{i j}\left(\lambda_{a b d}^{L} L_{a}^{i} L_{b}^{j} E_{d}^{c}+\lambda_{a b d}^{L \prime} L_{a}^{i} Q_{b}^{j} D_{d}^{c}+\mu_{a}^{\prime} L_{a}^{i} H_{2}^{j}\right)+\lambda_{a b d}^{B} U_{a}^{c} D_{b}^{c} D_{d}^{c} .
$$

These terms are absent in the SM. The reason is very simple: one can not replace the superfields in Eqn. (2.4) by the ordinary fields like in Eqn. (2.3) because of the Lorentz invariance. These terms have also another property, they violate either the lepton number $L$ (the first 3 terms in Eqn. (2.4)) 
or the baryon number $B$ (the last term). Since both effects are not observed in Nature, these terms must be suppressed or excluded. One can avoid such terms introducing a new special symmetry called $R$-symmetry [22]. This is the global $U(1)_{R}$ invariance

$$
U(1)_{R}: \quad \theta \rightarrow e^{i \alpha} \theta, \Phi \rightarrow e^{i n \alpha} \Phi,
$$

which is reduced to the discrete group $Z_{2}$ called $R$-parity. The $R$-parity quantum number is

$$
R=(-1)^{3(B-L)+2 S}
$$

for the particles with the spin $S$. Thus, all the ordinary particles have the $R$-parity quantum number equal to $R=+1$, while all the superpartners have the $R$-parity quantum number equal to $R=-1$. The first part of the Yukawa Lagrangian is $R$-symmetric, while the second part is $R$-nonsymmetric. The $R$-parity obviously forbids the terms (2.4). However, it may well be that these terms are present, though experimental limits on the couplings are very severe

$$
\lambda_{a b c}^{L}, \quad \lambda_{a b c}^{L \prime}<10^{-4}, \quad \lambda_{a b c}^{B}<10^{-9} .
$$

Conservation of the $R$-parity has two important consequences

- the superpartners are created in pairs;

- the lightest superparticle (LSP) is stable. Usually it is the photino $\tilde{\gamma}$, the superpartner of the photon with some admixture of the neutral higgsino. This is the candidate for the DM particle which should be neutral and survive since the Big Bang.

\subsection{Next-to-Minimal Supersymmetry}

Some problems of the MSSM are sometimes proposed to be solved within its minimal extension which is called the Next-to-Minimal Supersymmetric Standard Model (NMSSM) [23]. It contains additional scalar superfield $S$ which is singlet with respect to the SM symmetry group. Enlarging the Higgs sector ( 7 instead of 5 Higgs bosons) this theory allows to smouth the problem of the lightest Higgs boson mass and to explain why the Higgs mixing parameter $\mu$ is around 100 $\mathrm{GeV}$.

The superpotential of the NMSSM contains few additional terms

$$
\Delta \mathscr{L}=\lambda S H_{1} H_{2}+\frac{\kappa}{3} S^{3}+m_{s}^{2} S^{2}
$$

with arbitrary parameters. Being expanded in components the scalarsuper field $S$ gives two additional neutral Higgses (one scalar and one pseudoscalar ) and the corresponding higgsinos. The vacuum expectation values of the singlet field $\langle s>$ leads to the Higgs mixing term with $\mu=\lambda\langle s\rangle$. One has also the corresponding soft terms. The main features of the NMSSM are similar to the MSSM except for the Higgs and neutralino sectors.

\subsection{Properties of interactions}

If one assumes that the $R$-parity is preserved, then the interactions of the superpartners are essentially the same as in the SM, but two of three particles involved into the interaction at any vertex are replaced by the superpartners. The reason for it is the $R$-parity.

Typical vertices are shown in Fig. 7. The tilde above the letter denotes the corresponding superpartner. Note that the coupling is the same in all the vertices involving the superpartners. 


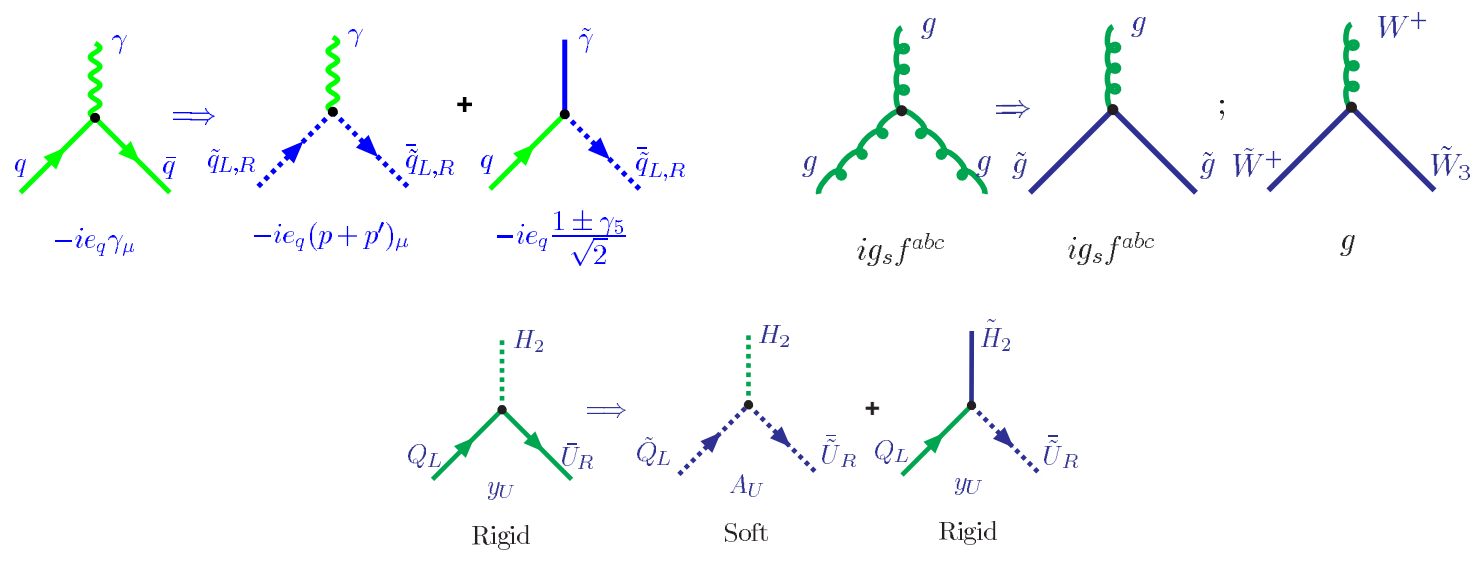

Figure 7: The gauge-matter interaction, the gauge self-interaction and the Yukawa interaction.

\subsection{Creation and decay of superpartners}

The above-mentioned rule together with the Feynman rules for the SM enables one to draw diagrams describing creation of the superpartners. One of the most promising processes is the $e^{+} e^{-}$ annihilation (see Fig. 8).

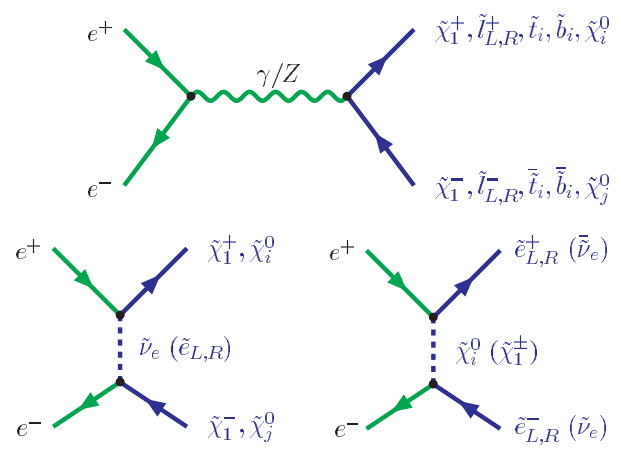

Figure 8: Creation of the superpartners at electron-positron colliders.

The usual kinematic restriction is given by the c.m. energy $m_{\text {sparticle }}^{\max } \leq \sqrt{s} / 2$. Similar processes take place at hadron colliders with the electrons and the positrons being replaced by the quarks and the gluons.

Experimental signatures at the hadron colliders are similar to those at the $e^{+} e^{-}$machines; however, here one has wider possibilities. Besides the usual annihilation channel, one has numerous processes of gluon fusion, quark-antiquark and quark-gluon scattering (see Fig. 9).

Creation of the superpartners can be accompanied by creation of the ordinary particles as well. We consider various experimental signatures below. They crucially depend on the SUSY breaking pattern and on the mass spectrum of the superpartners.

The decay properties of the superpartners also depend on their masses. For the quark and lepton superpartners the main processes are shown in Fig. 10. 

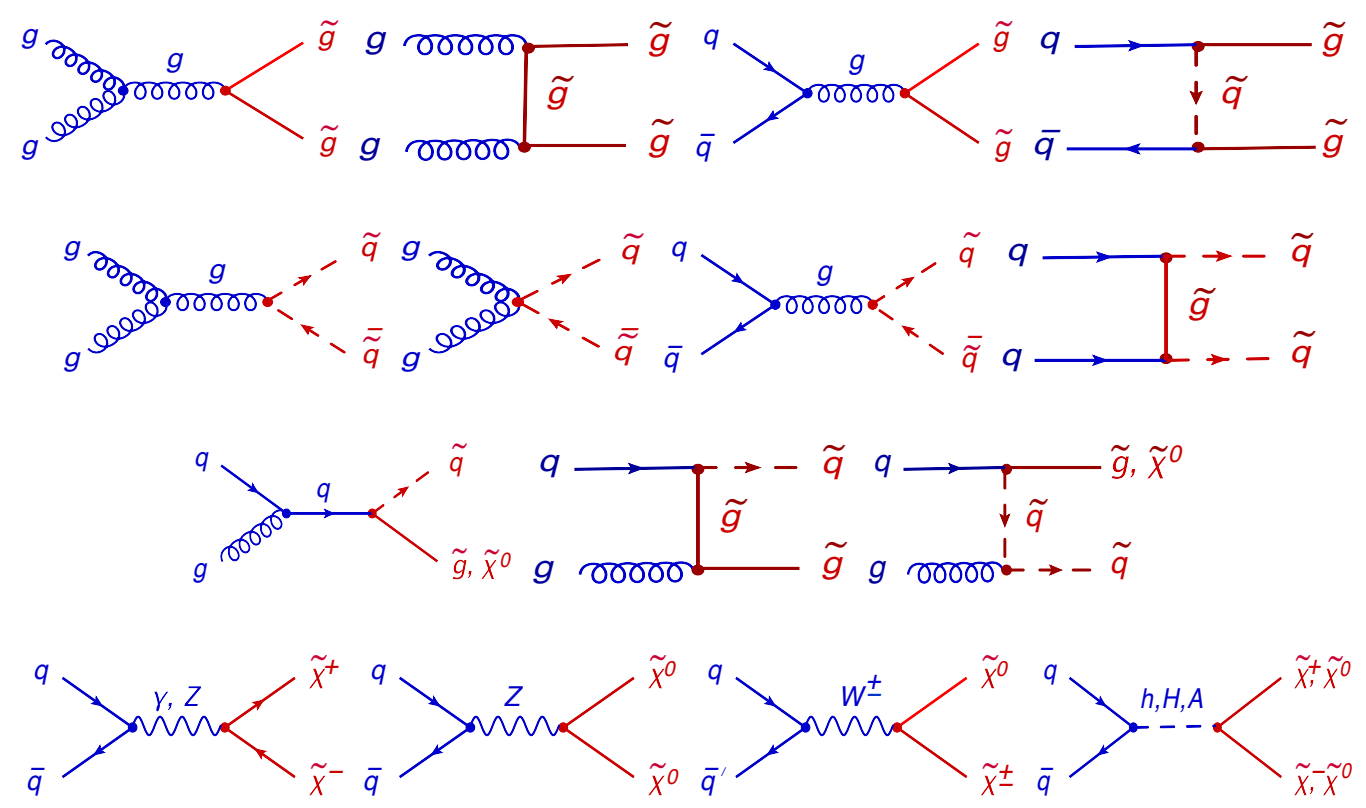

Figure 9: Examples of diagrams for the SUSY particle production via the strong interactions (top rows for $\tilde{g} \tilde{g}, \tilde{q} \tilde{\tilde{q}}$ and $\tilde{g} \tilde{q}$, respectively) and the electroweak interactions (the lowest row).

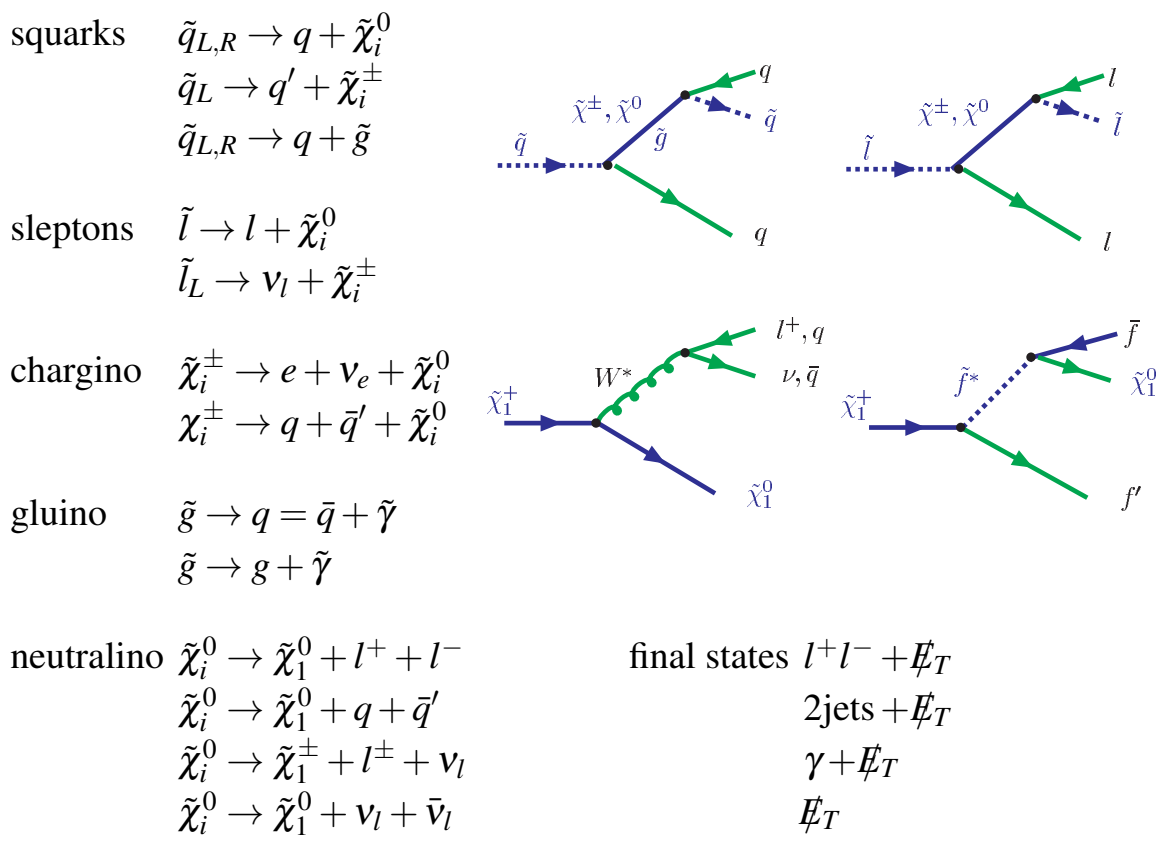

Figure 10: Decay of superpartners 


\section{Breaking of SUSY in the MSSM}

Usually it is assumed that the supersymmetry is broken spontaneously via the v.e.v.s of some fields. However, in the case of supersymmetry one can not use the scalar fields like the Higgs field, but rather the auxiliary fields present in any SUSY multiplet. There are two basic mechanisms of spontaneous SUSY breaking: the Fayet-Iliopoulos (or $D$-type) mechanism [9] based on the $D$ auxiliary field from the vector multiplet and the O'Raifeartaigh (or $F$-type) mechanism [10] based on the $F$ auxiliary field from the chiral multiplet. Unfortunately, one can not explicitly use these mechanisms within the MSSM since none of the fields of the MSSM can develop the non-zero v.e.v. without spoiling the gauge invariance. Therefore, the spontaneous SUSY breaking should take place via some other fields.

The most common scenario for producing low-energy supersymmetry breaking is called the hidden sector scenario [24]. According to this scenario, there exist two sectors: the usual matter belongs to the "visible" one, while the second, "hidden" sector, contains the fields which lead to breaking of the supersymmetry. These two sectors interact with each other by an exchange of some fields called messengers, which mediate SUSY breaking from the hidden to the visible sector. There might be various types of the messenger fields: gravity, gauge, etc. The hidden sector is the weakest part of the MSSM. It contains a lot of ambiguities and leads to uncertainties of the MSSM predictions considered below.

So far there are four known main mechanisms to mediate SUSY breaking from the hidden to the visible sector:

- Gravity mediation (SUGRA) [25];

- Gauge mediation [26];

- Anomaly mediation [27];

- Gaugino mediation [28].

All the four mechanisms of soft SUSY breaking are different in details but are common in results. The predictions for the sparticle spectrum depend on the mechanism of SUSY breaking. In what follows, to calculate the mass spectrum of the superpartners, we need the explicit form of the SUSY breaking terms. For the MSSM without the $R$-parity violation one has in general

$$
\begin{aligned}
& -\mathscr{L}_{\text {Breaking }}= \\
& =\sum_{i} m_{0 i}^{2}\left|\varphi_{i}\right|^{2}+\left(\frac{1}{2} \sum_{\alpha} M_{\alpha} \tilde{\lambda}_{\alpha} \tilde{\lambda}_{\alpha}+B H_{1} H_{2}+A_{a b}^{U} \tilde{Q}_{a} \tilde{U}_{b}^{c} H_{2}+A_{a b}^{D} \tilde{Q}_{a} \tilde{D}_{b}^{c} H_{1}+A_{a b}^{L} \tilde{L}_{a} \tilde{E}_{b}^{c} H_{1}\right),
\end{aligned}
$$

where we have suppressed the $S U(2)$ indices. Here $\varphi_{i}$ are all the scalar fields, $\tilde{\lambda}_{\alpha}$ are the gaugino fields, $\tilde{Q}, \tilde{U}, \tilde{D}$ and $\tilde{L}, \tilde{E}$ are the squark and slepton fields, respectively, and $H_{1,2}$ are the $\mathrm{SU}(2)$ doublet Higgs fields.

Eqn. (3.1) contains a vast number of free parameters which spoils the predictiive power of the model. To reduce their number, we adopt the so-called universality hypothesis, i. e., we assume 
the universality or equality of various soft parameters at the high energy scale, namely, we put all the spin- 0 particle masses to be equal to the universal value $m_{0}$, all the spin-1/2 particle (gaugino) masses to be equal to $m_{1 / 2}$ and all the cubic and quadratic terms, proportional to $A$ and $B$, to repeat the structure of the Yukawa superpotential (2.3). This is the additional requirement motivated by the supergravity mechanism of SUSY breaking. The universality is not the necessary requirement and one may consider the non-universal soft terms as well. However, it will not change the qualitative picture presented below; so, for simplicity, in what follows we consider the universal boundary conditions. In this case, Eqn. (3.1) takes the form

$$
\begin{aligned}
& -\mathscr{L}_{\text {Breaking }}= \\
& =m_{0}^{2} \sum_{i}\left|\varphi_{i}\right|^{2}+\left(\frac{m_{1 / 2}}{2} \sum_{\alpha} \tilde{\lambda}_{\alpha} \tilde{\lambda}_{\alpha}+B \mu H_{1} H_{2}+A\left[y_{a b}^{U} \tilde{Q}_{a} \tilde{U}_{b}^{c} H_{2}+y_{a b}^{D} \tilde{Q}_{a} \tilde{D}_{b}^{c} H_{1}+y_{a b}^{L} \tilde{L}_{a} \tilde{E}_{b}^{c} H_{1}\right]\right) .
\end{aligned}
$$

Thus, we are left with five free parameters, namely, $m_{0}, m_{1 / 2}, A, B$ and $\mu$ versus two parameters of the Higgs potential in the SM, $m^{2}$ and $\lambda$. In the SUSY model the Higgs potential is not arbitrary but is calculated from the Yukawa and gauge terms as we will see below.

The soft terms explicitly break the supersymmetry. As will be shown later, they lead to the mass spectrum of the superpartners different from that of the ordinary particles. Remind that the masses of the quarks and leptons remain zero until the $S U(2)$ symmetry is spontaneously broken.

\subsection{The soft terms and the mass formulae}

There are two main sources of the mass terms in the Lagrangian: the $D$-terms and the soft ones. With given values of $m_{0}, m_{1 / 2}, \mu, Y_{t}, Y_{b}, Y_{\tau}, A$, and $B$ one can construct the mass matrices for all the particles. Knowing them at the GUT scale, one can solve the corresponding RG equations, thus linking the values at the GUT and electroweak scales. Substituting these parameters into the mass matrices, one can predict the mass spectrum of the superpartners $[29,30]$.

\subsubsection{Gaugino-higgsino mass terms}

The mass matrix for the gauginos, the superpartners of the gauge bosons, and for the higgsinos, the superpartners of the Higgs bosons, is nondiagonal, thus leading to their mixing. The mass terms look like

$$
\mathscr{L}_{\text {Gaugino-Higgsino }}=-\frac{1}{2} M_{3} \bar{\lambda}_{a} \lambda_{a}-\frac{1}{2} \bar{\chi} M^{(0)} \chi-\left(\bar{\psi} M^{(c)} \psi+\text { h.c. }\right),
$$

where $\lambda_{a}, a=1,2, \ldots, 8$ are the Majorana gluino fields and

$$
\chi=\left(\begin{array}{c}
\tilde{B}^{0} \\
\tilde{W}^{3} \\
\tilde{H}_{1}^{0} \\
\tilde{H}_{2}^{0}
\end{array}\right), \quad \psi=\left(\begin{array}{c}
\tilde{W}^{+} \\
\tilde{H}^{+}
\end{array}\right)
$$

are, respectively, the Majorana neutralino and the Dirac chargino fields.

The neutralino mass matrix is

$$
M^{(0)}=\left(\begin{array}{cccc}
M_{1} & 0 & -M_{Z} \cos \beta \sin \theta_{W} & M_{Z} \sin \beta \sin \theta_{W} \\
0 & M_{2} & M_{Z} \cos \beta \cos \theta_{W} & -M_{Z} \sin \beta \cos \theta_{W} \\
-M_{Z} \cos \beta \sin \theta_{W} & M_{Z} \cos \beta \cos \theta_{W} & 0 & -\mu \\
M_{Z} \sin \beta \sin \theta_{W} & -M_{Z} \sin \beta \cos \theta_{W} & -\mu & 0
\end{array}\right)
$$


where $\tan \beta=v_{2} / v_{1}$ is the ratio of two Higgs v.e.v.s and $\sin \theta_{W}$ is the usual sine of the weak mixing angle. The physical neutralino masses $M_{\tilde{\chi}_{i}^{0}}$ are obtained as eigenvalues of this matrix after diagonalization.

For the chargino mass matrix one has

$$
M^{(c)}=\left(\begin{array}{cc}
M_{2} & \sqrt{2} M_{W} \sin \beta \\
\sqrt{2} M_{W} \cos \beta & \mu
\end{array}\right) .
$$

This matrix has two chargino eigenstates $\tilde{\chi}_{1,2}^{ \pm}$with mass eigenvalues

$$
M_{1,2}^{2}=\frac{1}{2}\left[M_{2}^{2}+\mu^{2}+2 M_{W}^{2} \mp \sqrt{\left(M_{2}^{2}-\mu^{2}\right)^{2}+4 M_{W}^{4} \cos ^{2} 2 \beta+4 M_{W}^{2}\left(M_{2}^{2}+\mu^{2}+2 M_{2} \mu \sin 2 \beta\right)}\right] .
$$

\subsubsection{Squark and slepton masses}

The non-negligible Yukawa couplings cause mixing between the electroweak eigenstates and the mass eigenstates of the third generation particles. The mixing matrices for $\tilde{m}_{t}^{2}, \tilde{m}_{b}^{2}$ and $\tilde{m}_{\tau}^{2}$ are

$$
\begin{gathered}
\left(\begin{array}{cc}
\tilde{m}_{t L}^{2} & m_{t}\left(A_{t}-\mu \cot \beta\right) \\
m_{t}\left(A_{t}-\mu \cot \beta\right) & \tilde{m}_{t R}^{2}
\end{array}\right), \\
\left(\begin{array}{cc}
\tilde{m}_{b L}^{2} & m_{b}\left(A_{b}-\mu \tan \beta\right) \\
m_{b}\left(A_{b}-\mu \tan \beta\right) & \tilde{m}_{b R}^{2}
\end{array}\right), \\
\left(\begin{array}{cc}
\tilde{m}_{\tau L}^{2} & m_{\tau}\left(A_{\tau}-\mu \tan \beta\right) \\
m_{\tau}\left(A_{\tau}-\mu \tan \beta\right) & \tilde{m}_{\tau R}^{2}
\end{array}\right)
\end{gathered}
$$

with

$$
\begin{aligned}
& \tilde{m}_{t L}^{2}=\tilde{m}_{Q}^{2}+m_{t}^{2}+\frac{1}{6}\left(4 M_{W}^{2}-M_{Z}^{2}\right) \cos 2 \beta, \\
& \tilde{m}_{t R}^{2}=\tilde{m}_{U}^{2}+m_{t}^{2}-\frac{2}{3}\left(M_{W}^{2}-M_{Z}^{2}\right) \cos 2 \beta, \\
& \tilde{m}_{b L}^{2}=\tilde{m}_{Q}^{2}+m_{b}^{2}-\frac{1}{6}\left(2 M_{W}^{2}+M_{Z}^{2}\right) \cos 2 \beta, \\
& \tilde{m}_{b R}^{2}=\tilde{m}_{D}^{2}+m_{b}^{2}+\frac{1}{3}\left(M_{W}^{2}-M_{Z}^{2}\right) \cos 2 \beta, \\
& \tilde{m}_{\tau L}^{2}=\tilde{m}_{L}^{2}+m_{\tau}^{2}-\frac{1}{2}\left(2 M_{W}^{2}-M_{Z}^{2}\right) \cos 2 \beta, \\
& \tilde{m}_{\tau R}^{2}=\tilde{m}_{E}^{2}+m_{\tau}^{2}+\left(M_{W}^{2}-M_{Z}^{2}\right) \cos 2 \beta
\end{aligned}
$$

and the mass eigenstates are the eigenvalues of these mass matrices. For the light generations mixing is negligible.

The first terms here $\left(\tilde{m}^{2}\right)$ are the soft ones, which are calculated using the RG equations starting from their values at the GUT (Planck) scale. The second ones are the usual masses of the quarks and leptons and the last ones are the $D$-terms of the potential. 


\subsection{The Higgs potential}

As has already been mentioned, the Higgs potential in the MSSM is totally defined by the superpotential (and the soft terms). Due to the structure of $\mathscr{L}_{\text {Yukawa }}$ the Higgs self-interaction is given by the $D$-terms while the $F$-terms contribute only to the mass matrix. The tree level potential is

$$
V_{\text {tree }}=m_{1}^{2}\left|H_{1}\right|^{2}+m_{2}^{2}\left|H_{2}\right|^{2}-m_{3}^{2}\left(H_{1} H_{2}+\text { h.c. }\right)+\frac{g^{2}+g^{\prime 2}}{8}\left(\left|H_{1}\right|^{2}-\left|H_{2}\right|^{2}\right)^{2}+\frac{g^{2}}{2}\left|H_{1}^{*} H_{2}\right|^{2},
$$

where $m_{1}^{2}=m_{H_{1}}^{2}+\mu^{2}, m_{2}^{2}=m_{H_{2}}^{2}+\mu^{2}$. At the GUT scale $m_{1}^{2}=m_{2}^{2}=m_{0}^{2}+\mu_{0}^{2}, m_{3}^{2}=-B \mu_{0}$. Notice that the Higgs self-interaction coupling in Eqn. (3.6) is fixed and defined by the gauge interactions as opposed to the Standard Model.

The Higgs scalar potential in accordance with the supersymmetry, is positive definite and stable. It has no nontrivial minimum different from zero. Indeed, let us write the minimization condition for the potential (3.6)

$$
\begin{aligned}
& \frac{1}{2} \frac{\delta V}{\delta H_{1}}=m_{1}^{2} v_{1}-m_{3}^{2} v_{2}+\frac{g^{2}+g^{\prime 2}}{4}\left(v_{1}^{2}-v_{2}^{2}\right) v_{1}=0, \\
& \frac{1}{2} \frac{\delta V}{\delta H_{2}}=m_{2}^{2} v_{2}-m_{3}^{2} v_{1}+\frac{g^{2}+g^{\prime 2}}{4}\left(v_{1}^{2}-v_{2}^{2}\right) v_{2}=0
\end{aligned}
$$

where we have introduced the notation

$$
\begin{gathered}
\left\langle H_{1}\right\rangle \equiv v_{1}=v \cos \beta, \quad\left\langle H_{2}\right\rangle \equiv v_{2}=v \sin \beta, \\
v^{2}=v_{1}^{2}+v_{2}^{2}, \quad \tan \beta \equiv \frac{v_{2}}{v_{1}} .
\end{gathered}
$$

Solution to Eqs. (3.7) can be expressed in terms of $v^{2}$ and $\sin 2 \beta$

$$
v^{2}=\frac{4\left(m_{1}^{2}-m_{2}^{2} \tan ^{2} \beta\right)}{\left(g^{2}+g^{\prime 2}\right)\left(\tan ^{2} \beta-1\right)}, \quad \sin 2 \beta=\frac{2 m_{3}^{2}}{m_{1}^{2}+m_{2}^{2}} .
$$

One can easily see from Eqn. (3.8) that if $m_{1}^{2}=m_{2}^{2}=m_{0}^{2}+\mu_{0}^{2}, v^{2}$ happens to be negative, i. e. the minimum does not exist. In fact, real positive solutions to Eqs. (3.7) exist only if the following conditions are satisfied:

$$
m_{1}^{2}+m_{2}^{2}>2 m_{3}^{2}, \quad m_{1}^{2} m_{2}^{2}<m_{3}^{4},
$$

which is not the case at the GUT scale. This means that spontaneous breaking of the $S U(2)$ gauge invariance, which is needed in the SM to give masses for all the particles, does not take place in the MSSM.

This strong statement is valid, however, only at the GUT scale. Indeed, going down with the energy, the parameters of the potential (3.6) are renormalized. They become the "running" parameters with the energy scale dependence given by the RG equations. 


\subsection{Radiative electroweak symmetry breaking}

The running of the Higgs masses leads to the remarkable phenomenon known as radiative electroweak symmetry breaking. Indeed, one can see in Fig. 11 that $m_{2}^{2}$ (or both $m_{1}^{2}$ and $m_{2}^{2}$ ) decreases when going down from the GUT scale to the $M_{Z}$ scale and can even become negative. As a result, at some value of $Q^{2}$ the conditions (3.9) are satisfied, so that the nontrivial minimum appears. This triggers spontaneous breaking of the $S U(2)$ gauge invariance as shown in Fig.5 above. The vacuum expectations of the Higgs fields acquire nonzero values and provide masses to the quarks, leptons and $S U(2)$ gauge bosons, and additional contributions to the masses of their superpartners.

In this way one also obtains the explanation of why the two scales are so much different. Due to the logarithmic running of the parameters, one needs a long "running time" to get $m_{2}^{2}$ (or both $m_{1}^{2}$ and $m_{2}^{2}$ ) to be negative when starting from a positive value of the order of $M_{S U S Y} \sim 10^{2} \div 10^{3} \mathrm{GeV}$ at the GUT scale.
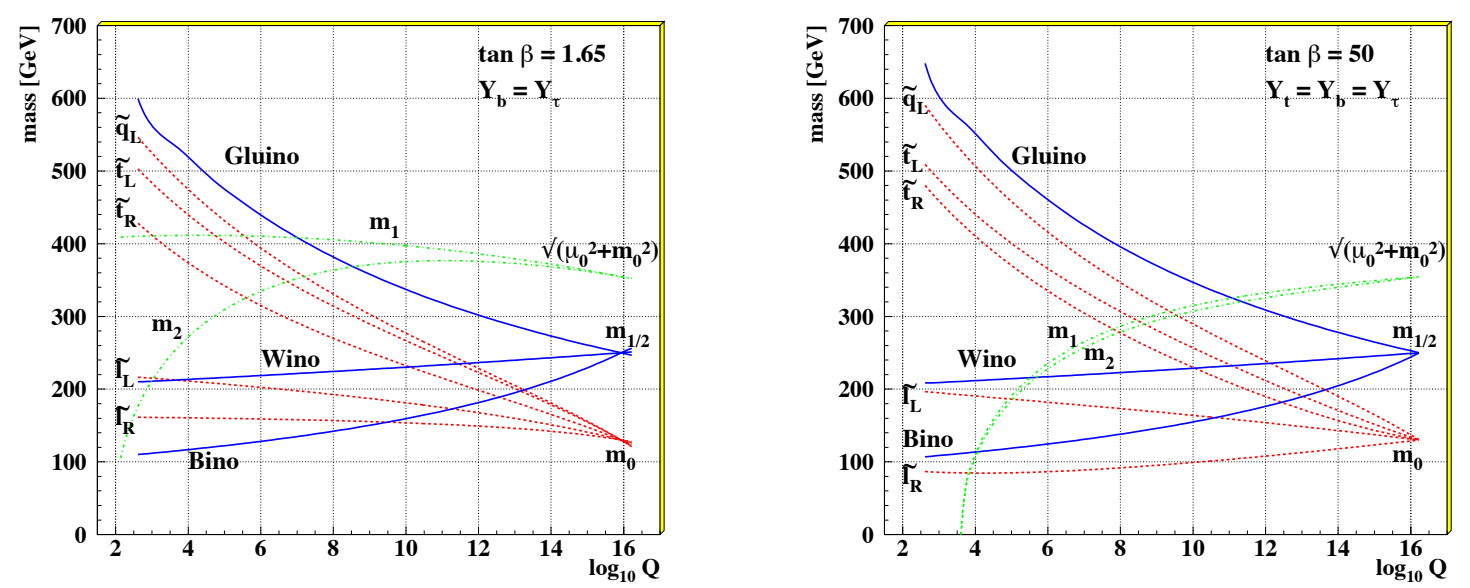

Figure 11: An example of evolution of superparticle masses and soft supersymmetry breaking parameters $m_{1}^{2}=m_{H_{1}}^{2}+\mu^{2}$ and $m_{2}^{2}=m_{H_{2}}^{2}+\mu^{2}$ for low (left) and high (right) values of $\tan \beta$.

\subsection{The superpartners mass spectrum}

The mass spectrum is defined by the low energy parameters. To calculate the low energy values of the soft terms, we use the corresponding RG equations [31]. Having all the RG equations, one can now find the RG flow for the soft terms. Taking the initial values of the soft masses at the GUT scale in the interval between $10^{2} \div 10^{3} \mathrm{GeV}$ consistent with the SUSY scale suggested by the unification of the gauge couplings (see Fig.3) leads to the RG flow of the soft terms shown in Fig. 11. [29, 30]

One should mention the following general features common to any choice of initial conditions:

- The gaugino masses follow the running of the gauge couplings and split at low energies. The gluino mass is running faster than the other ones and is usually the heaviest due to the strong interaction.

- The squark and slepton masses also split at low energies, the stops (and sbottoms) being the lightest due to the relatively big Yukawa couplings of the third generation. 
- The Higgs masses (or at least one of them) are running down very quickly and may even become negative.

The typical dependence of the mass spectra on the initial conditions at the GUT scale $\left(m_{0}\right)$ is also shown in Fig. 12 [32,33]. For a given value of $m_{1 / 2}$ the masses of the lightest particles are practically independent of $m_{0}$, while the masses of the heavier ones increase with it monotonically. One can see that the lightest neutralinos and charginos as well as the top-squark may be rather light.
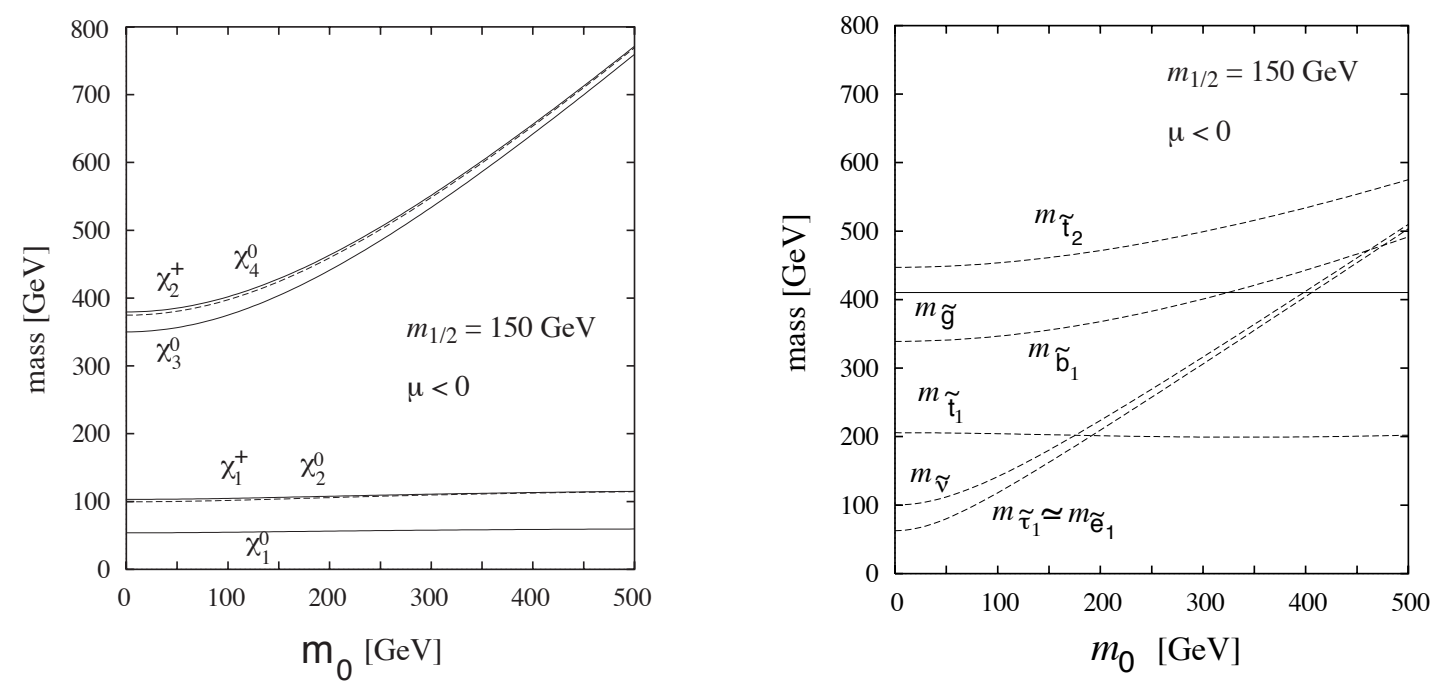

Figure 12: The masses of sparticles as functions of the initial value $m_{0}$.

\subsection{The Higgs boson masses}

Provided conditions (3.9) are satisfied, one can also calculate the masses of the Higgs bosons taking the second derivatives of the potential (3.6) with respect to the real and imaginary parts of the Higgs fields $\left(H_{i}=S_{i}+i P_{i}\right)$ in the minimum. The mass matrices at the tree level are

$C P$-odd components $P_{1}$ and $P_{2}$ :

$$
\mathscr{M}^{\text {odd }}=\left.\frac{\partial^{2} V}{\partial P_{i} \partial P_{j}}\right|_{H_{i}=v_{i}}=\left(\begin{array}{cc}
\tan \beta & 1 \\
1 & \cot \beta
\end{array}\right) m_{3}^{2},
$$

$C P$-even neutral components $S_{1}$ and $S_{2}$ :

$$
\mathscr{M}^{\text {even }}=\left.\frac{\partial^{2} V}{\partial S_{i} \partial S_{j}}\right|_{H_{i}=v_{i}}=\left(\begin{array}{cc}
\tan \beta & -1 \\
-1 & \cot \beta
\end{array}\right) m_{3}^{2}+\left(\begin{array}{cc}
\cot \beta & -1 \\
-1 & \tan \beta
\end{array}\right) M_{Z}^{2} \frac{\sin 2 \beta}{2},
$$

Charged components $H^{-}$and $H^{+}$:

$$
\mathscr{M}^{c h}=\left.\frac{\partial^{2} V}{\partial H_{i}^{+} \partial H_{j}^{-}}\right|_{H_{i}=v_{i}}=\left(\begin{array}{cc}
\tan \beta & 1 \\
1 & \cot \beta
\end{array}\right)\left(m_{3}^{2}+M_{W}^{2} \frac{\sin 2 \beta}{2}\right) .
$$


Diagonalizing the mass matrices, one gets the mass eigenstates:

$$
\begin{gathered}
\left\{\begin{array}{l}
G^{0}=-\cos \beta P_{1}+\sin \beta P_{2}, \text { Goldstone boson } \rightarrow Z_{0}, \\
A=\sin \beta P_{1}+\cos \beta P_{2}, \text { Neutral } C P-\text { odd Higgs, }
\end{array}\right. \\
\left\{\begin{array}{l}
G^{+}=-\cos \beta\left(H_{1}^{-}\right)^{*}+\sin \beta H_{2}^{+}, \text {Goldstone boson } \rightarrow W^{+}, \\
H^{+}=\sin \beta\left(H_{1}^{-}\right)^{*}+\cos \beta H_{2}^{+}, \text {Charged Higgs, }
\end{array}\right. \\
\left\{\begin{array}{l}
h=-\sin \alpha S_{1}+\cos \alpha S_{2}, \text { SM CP-even Higgs, } \\
H=\cos \alpha S_{1}+\sin \alpha S_{2}, \text { Extra heavy Higgs, }
\end{array}\right.
\end{gathered}
$$

where the mixing angle $\alpha$ is given by

$$
\tan 2 \alpha=\tan 2 \beta\left(\frac{m_{A}^{2}+M_{Z}^{2}}{m_{A}^{2}-M_{Z}^{2}}\right) .
$$

The physical Higgs bosons acquire the following masses [20]:

$C P$-odd neutral Higgs $A$ :

$$
m_{A}^{2}=m_{1}^{2}+m_{2}^{2},
$$

Charged Higgses $H^{ \pm}$:

$$
m_{H^{ \pm}}^{2}=m_{A}^{2}+M_{W}^{2}
$$

$C P$-even neutral Higgses $H, h$ :

$$
m_{H, h}^{2}=\frac{1}{2}\left[m_{A}^{2}+M_{Z}^{2} \pm \sqrt{\left(m_{A}^{2}+M_{Z}^{2}\right)^{2}-4 m_{A}^{2} M_{Z}^{2} \cos ^{2} 2 \beta}\right],
$$

where, as usual,

$$
M_{W}^{2}=\frac{g^{2}}{2} v^{2}, \quad M_{Z}^{2}=\frac{g^{2}+g^{\prime 2}}{2} v^{2} .
$$

This leads to the once celebrated SUSY mass relations

$$
\begin{gathered}
m_{H^{ \pm}} \geq M_{W}, \quad m_{h} \leq m_{A} \leq M_{H}, \\
m_{h} \leq M_{Z}|\cos 2 \beta| \leq M_{Z}, \\
m_{h}^{2}+m_{H}^{2}=m_{A}^{2}+M_{Z}^{2} .
\end{gathered}
$$

Thus, the lightest neutral Higgs boson happens to be lighter than the Z-boson, which clearly distinguishes it from the SM one. Though we do not know the mass of the Higgs boson in the $\mathrm{SM}$, there are several indirect constraints leading to the lower boundary of $m_{h}^{S M} \geq 135 \mathrm{GeV}$. After including the leading one-loop radiative corrections, the mass of the lightest Higgs boson in the MSSM, $m_{h}$, reads

$$
m_{h}^{2}=M_{Z}^{2} \cos ^{2} 2 \beta+\frac{3 g^{2} m_{t}^{4}}{16 \pi^{2} M_{W}^{2}} \log \frac{\tilde{m}_{t_{1}}^{2} \tilde{m}_{t_{2}}^{2}}{m_{t}^{4}}+\ldots
$$

which leads to about $40 \mathrm{GeV}$ increase [34]. The second loop correction is negative but small [35]. 
It is interesting, that the Higgs mass upper bound depends crucially on some parameters of the model, and is almost independent on the choice of the other parameters. For example, the $1 \mathrm{GeV}$ change in the mass of the top quark leads to the $\sim 1 \mathrm{GeV}$ change in the Higgs mass upper bound. The dependence of the maximal Higgs mass on the supersymmetry breaking scale $M_{S}$ is shown in the left panel of Fig. 13 [36] for different scenarios of SUSY breaking. The widths of bands corresponds to the variation of the top mass in the range $170-176 \mathrm{GeV}$.
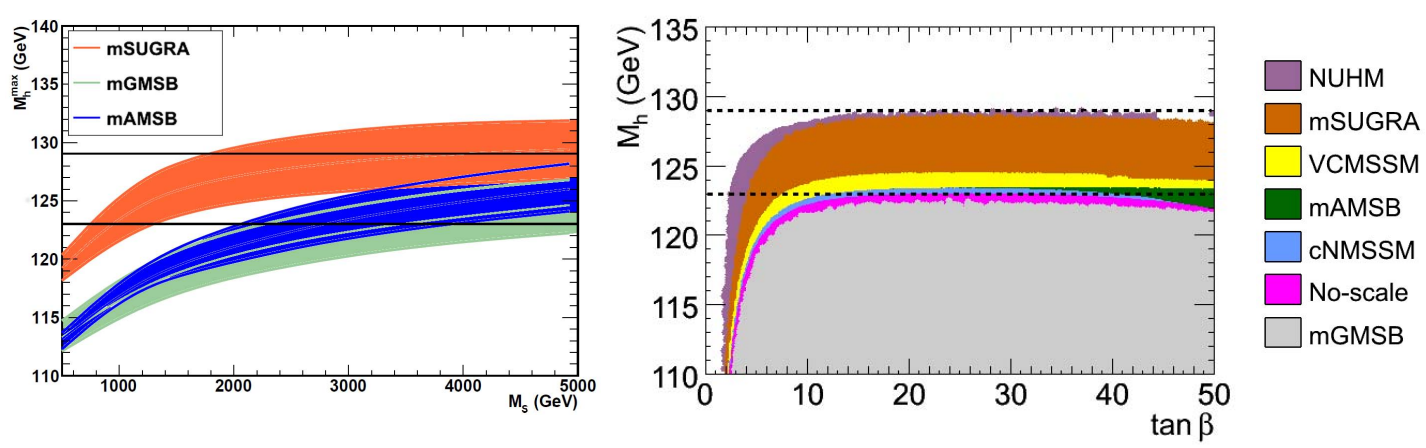

Figure 13: The maximal Higgs mass in the constrained MSSM scenarios mSUGRA, mAMSB and mGMSB, as a function of the scale $M_{S}$ when the top quark mass is varied in the range $m_{t}=170-176 \mathrm{GeV}$ (left) and as a function of $\tan \beta$ (right).

The right panel of Fig. 13 shows the dependence of the maximal Higgs mass on $\tan \beta$ for the fixed value of $m_{t}=173 \mathrm{GeV}$ while other parameters of the model vary within the ranges [37]:

$$
\begin{aligned}
& \text { mSUGRA: } \quad 50 \mathrm{GeV} \leq m_{0} \leq 3 \mathrm{TeV}, \quad 50 \mathrm{GeV} \leq m_{1 / 2} \leq 3 \mathrm{TeV}, \quad\left|A_{0}\right| \leq 9 \mathrm{TeV} ; \\
& \text { GMSB: } \quad 10 \mathrm{TeV} \leq \Lambda \leq 1000 \mathrm{TeV}, \quad 1 \leq M_{\text {mess }} / \Lambda \leq 10^{11}, \quad N_{\text {mess }}=1 \text {; } \\
& \text { AMSB: } \quad 1 \mathrm{TeV} \leq m_{3 / 2} \leq 100 \mathrm{TeV}, \quad 50 \mathrm{GeV} \leq m_{0} \leq 3 \mathrm{TeV} .
\end{aligned}
$$

New possibilities appear if one enlarges the Higgs sector. The next popular step is the introduction of an additional Higgs field which is a singlet with respect to the gauge group of the SM, i.e. transition from the MSSM to the NMSSM [23]. Here one has already seven Higgs bosons and three of them CP-even and neutral. The novel feature of this model is that already at the tree level Eqn.(3.17) is modified by additional term

$$
m_{h}^{2}=M_{Z}^{2} \cos ^{2} 2 \beta+\lambda^{2} v^{2} \sin ^{2} 2 \beta+\ldots
$$

thus, allowing for higher values of the Higgs mass. This allows one to relax the slight tension which appears in the Constrained MSSM with respect to the $125 \mathrm{GeV}$ Higgs boson. The sample spectrum of particles for various models is shown in Fig.14 (right).

Note that in the case of the NMSSM one has two light CP-even Higgs bosons and the discovered particle with the mass of $125 \mathrm{GeV}$ might correspond to both $H_{1}$ and to $H_{2}$. The reason why we do not see the lightest Higgs boson $H_{1}$ in the second case is that it has a large admixture of the singlet state and hence very weakly interacts with the SM particles. 


\begin{tabular}{|c|c|}
\hline Model & Particle content \\
\hline SM & $\mathrm{h}$ CP-even \\
\hline 2HDM/MSSM & $\begin{array}{c}\mathrm{h}, \mathrm{H} \text { CP-even } \\
\mathrm{A} \mathrm{CP} \text {-odd } \\
\mathrm{H}\end{array}$ \\
\hline NMSSM & $\begin{array}{c}\mathrm{H}_{1}, \mathrm{H}_{2}, \mathrm{H}_{3} \text { CP-even } \\
\mathrm{A}_{1}, \mathrm{~A}_{2} \text { CP-odd } \\
\mathrm{H}\end{array}$ \\
\hline Composite & $\begin{array}{c}\mathrm{h} \text { CP-even } \\
+ \text { excited states }\end{array}$ \\
\hline
\end{tabular}

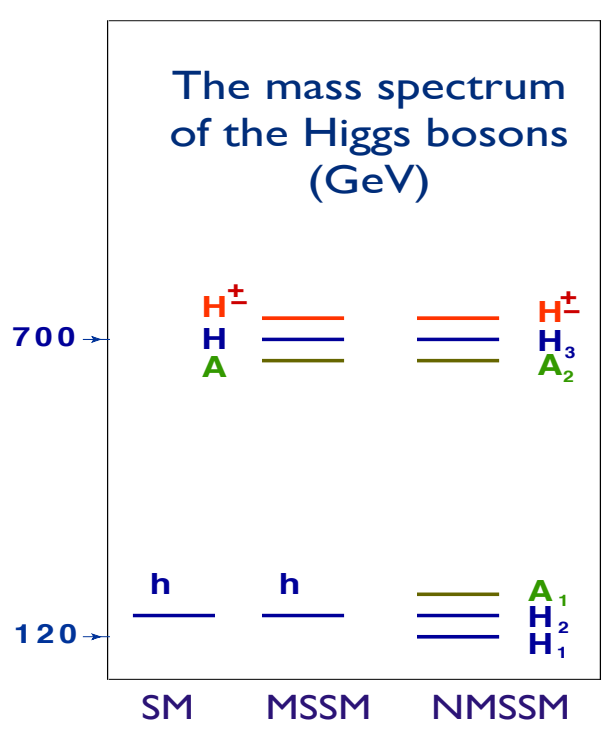

Figure 14: The field content and the spectrum in various models of the Higgs sector

\subsection{The lightest superparticle}

One of the crucial questions is the properties of the lightest superparticle. Different SUSY breaking scenarios lead to different experimental signatures and different LSP.

- Gravity mediation

In this case, the LSP is the lightest neutralino $\tilde{\chi}_{1}^{0}$, which is almost $90 \%$ photino for the low $\tan \beta$ solution and contains more higgsino admixture for high $\tan \beta$. The usual signature for LSP is the missing energy; $\tilde{\chi}_{1}^{0}$ is stable and is the best candidate for the cold dark matter particle in the Universe. Typical processes, where the LSP is created, end up with jets $+\not \phi_{T}$, or leptons $+\not \not_{T}$, or both jets + leptons $+\not \not_{T}$.

- Gauge mediation

In this case the LSP is the gravitino $\tilde{G}$, which also leads to the missing energy. The actual question here is what is the NLSP, the next-to-lightest particle, is. There are two possibilities:

i) $\tilde{\chi}_{1}^{0}$ is the NLSP. Then the decay modes are: $\tilde{\chi}_{1}^{0} \rightarrow \gamma \tilde{G}, h \tilde{G}, Z \tilde{G}$. As a result, one has two hard photons $+\not_{T}$, or jets $+\not_{T}$.

ii) $\tilde{l}_{R}$ is the NLSP. Then the decay mode is $\tilde{l}_{R} \rightarrow \tau \tilde{G}$ and the signature is a charged lepton and the missing energy.

- Anomaly mediation

In this case, one also has two possibilities:

i) $\tilde{\chi}_{1}^{0}$ is the LSP and wino-like. It is almost degenerate with the NLSP. 
ii) $\tilde{v}_{L}$ is the LSP. Then it appears in the decay of the chargino $\tilde{\chi}^{+} \rightarrow \tilde{v} l$ and the signature is the charged lepton and the missing energy.

- R-parity violation

In this case, the LSP is no longer stable and decays into the SM particles. It may be charged (or even colored) and may lead to rare decays like the neutrinoless double $\beta$-decay, etc.

Experimental limits on the LSP mass follow from the non-observation of the corresponding events. The modern lower limit is around $40 \mathrm{GeV}$.

\section{Where is SUSY?}

After many years of unsuccessful hunt for supersymmetry in particle physics experiments the natural question arises: where is supersymmetry? We try to answer this question searching for SUSY at accelerators, in the deep sky with the help of telescopes, and with the help of the underground facilities. It is obvious, that only direct detection of superpartners can convince people in discovery of supersymmetry, however combined information from the sky might give hints to the mass spectra and confirm the SUSY interpretation of the data.

\subsection{Search for SUSY at Colliders}

The strategy of the SUSY searches at colliders is based on the assumption that the masses of the superpartners indeed are in the region of $1 \mathrm{TeV}$ so that they might be created on the mass shell with the cross-section big enough to distinguish them from the background of the ordinary particles. Calculation of the background in the framework of the Standard Model thus becomes essential since the secondary particles in all the cases are the same.

There are many possibilities to create the superpartners at the hadron colliders. Besides the usual annihilation channel there are numerous processes of the gluon fusion, quark-antiquark and quark-gluon scattering. The maximal cross-sections of the order of a few picobarn can be achieved in the process of gluon fusion.

As a rule all the superpartners are short lived and decay into the ordinary particles and the lightest superparticle. The typical processes of creation of superpartners in strong and weak interaction are shown in Fig.15 [21]. The typical signature of supersymmetry is the presence of missing energy and missing transverse momentum carried away by the lightest supersymmetric particle $\chi_{1}^{0}$ which is neutral and stable.

The presence of missing energy and transverse momentum is the main difference from the background processes of the Standard Model. Contrary to the $e^{+} e^{-}$colliders, at hadron machines the background is extremely rich and essential. The missing energy is carried away by the heavy particle with the mass of the order of $100 \mathrm{GeV}$ that is essentially different from the processes with the neutrino in the final state. In hadron collisions the superpartners are always created in pairs and then further quickly decay creating a cascade with the ordinary quarks (i. e. hadron jets) or leptons in the final state plus the missing energy. For the case of the gluon fusion with the creation of gluino it is presented in Fig.15 (right panel). 

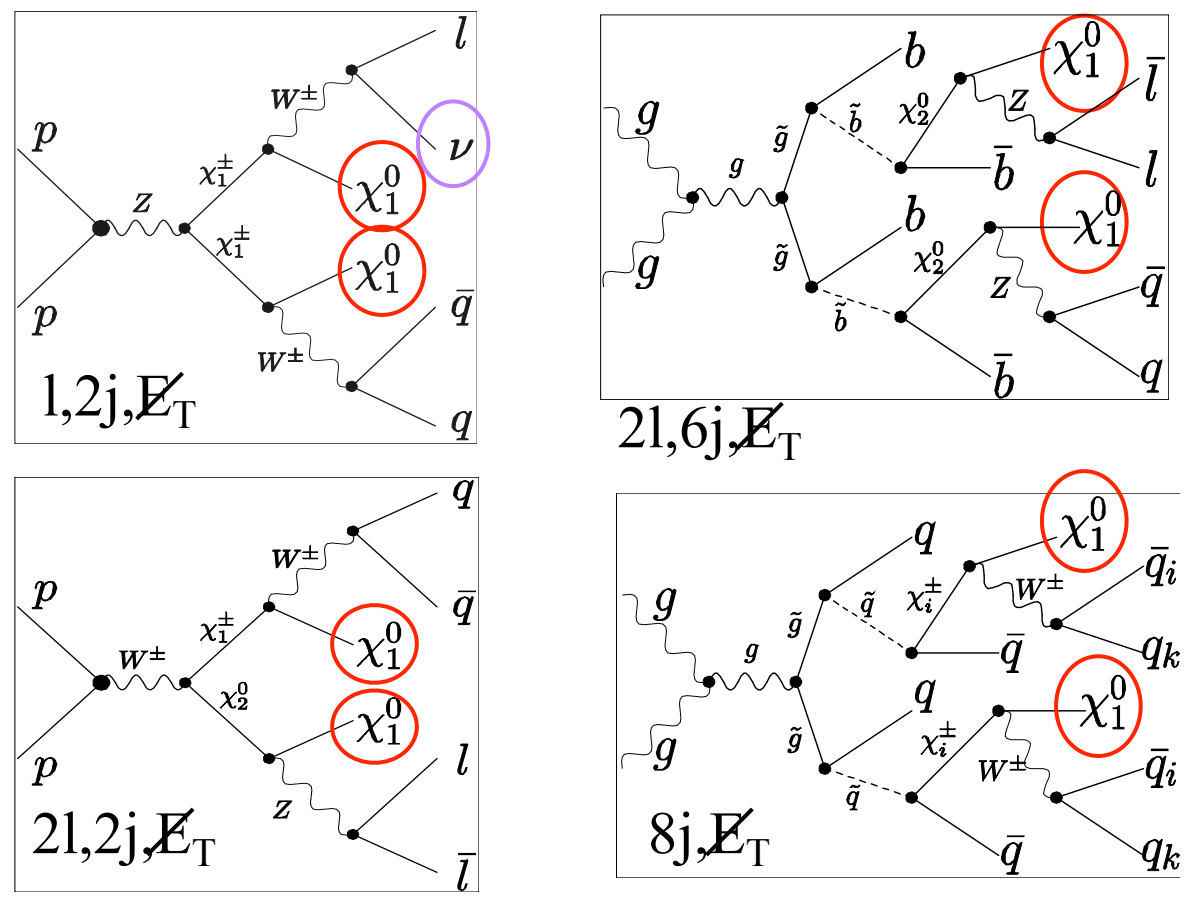

Figure 15: Creation of superpartners in weak (left) and strong (right) interactions. The expected final states are also shown. The particles escaping detection are encircled

The chargino and neutralino can also be produced in pairs through the Drell-Yang mechanism $p p \rightarrow \tilde{\chi}_{1}^{ \pm} \tilde{\chi}_{2}^{0}$ and can be detected via their lepton decays $\tilde{\chi}_{1}^{ \pm} \tilde{\chi}_{2}^{0} \rightarrow \ell \ell \ell+\not \phi_{T}$. Hence the main signal of their creation is the isolated leptons and the missing energy, see Fig.15(left panel). The main background in the trilepton channel comes from the creation of the standard particles $W Z / Z Z, t \bar{t}, Z b \bar{b}$ è $b \bar{b}$. There might be also the supersymmetric background from the cascade decays of the squarks and gluinos in multilepton modes.

So far the creation of superpartners at the LHC is not found, there are only limits on the masses of the hypothetical new particles. The boundary of possible values of masses of the scalar quarks and gluino have reached approximately 1500 and $1000 \mathrm{GeV}$, respectively. For the stop quarks it is almost two times lower. For the lightest neutralino the mass boundary varies between 100 and $400 \mathrm{GeV}$ depending on the values of the other masses. The constraints on the masses of charged weakly interacting particles almost two times higher than those for the neutral ones but depend on the decay mode. The obtained mass limits depend on the assumed decay modes which in their turn depend on the mass spectrum of superpartners, which is unknown. The presented constraints refer to the natural scenario.

\section{Conclusion}

Today after 40 years since the invention of supersymmetry we have no single convincing evidence that supersymmetry is realized in particle physics. Still it remains very popular in quantum 
field theory and in string theory due to its exceptional properties but needs experimental justification.

Let us remind the main pros and contras for supersymmetry in particle physics

Pro:

- Provides natural framework for unification with gravity

- Leads to gauge coupling unification (GUT)

- Solves the hierarchy problem

- Is a solid quantum field theory

- Provides natural candidate for the WIMP cold DM

- Predicts new particles and thus generates new job positions

Contra:

- Does not shed new light on the problem of

* Quark and lepton mass spectrum

* Quark and lepton mixing angles

$*$ the origin of $\mathrm{CP}$ violation

$*$ Number of flavours

* Baryon asymmetry of the Universe

- Doubles the number of particles

Low energy supersymmetry promises us that new physics is round the corner at the $\mathrm{TeV}$ scale to be exploited at colliders and astroparticle experiments of this decade. If our expectations are correct, very soon we will face new discoveries, the whole world of supersymmetric particles will show up and the table of fundamental particles will be enlarged in increasing rate. This would be a great step in understanding the microworld.

\section{Acknowledgements}

I would like to express my gratitude to the organizers of the Corfu School for their effort in creating a pleasant atmosphere and support. This work was partly supported by RFBR grant \# 14-02-00494.

\section{References}

[1] Y. A. Golfand and E. P. Likhtman, JETP Letters 13 (1971) 452;

D. V. Volkov and V. P. Akulov, JETP Letters 16 (1972) 621;

J. Wess and B. Zumino, Phys. Lett. B49 (1974) 52.

[2] P. Fayet and S. Ferrara, Phys. Rep. 32 (1977) 249;

M. F. Sohnius, Phys. Rep. 128 (1985) 41;

H. P. Nilles, Phys. Rep. 110 (1984) 1;

H. E. Haber and G. L. Kane, Phys. Rep. 117 (1985) 75;

A. B. Lahanas and D. V. Nanopoulos, Phys. Rep. 145 (1987) 1.

[3] J. Wess and J. Bagger, Supersymmetry and Supergravity, Princeton Univ. Press, 1983.

[4] A. Salam, J. Strathdee, Nucl. Phys. B76 (1974) 477;

S. Ferrara, J. Wess, B. Zumino, Phys. Lett. BS1 (1974) 239. 
[5] S. J. Gates, M. Grisaru, M. Roček and W. Siegel, Superspace or One Thousand and One Lessons in Supersymmetry, Benjamin \& Cummings, 1983;

P. West, Introduction to supersymmetry and supergravity, World Scientific, 1990;

S. Weinberg,The quantum theory of fields, Vol. 3, Cambridge, UK: Univ. Press, 2000.

[6] S. Coleman and J .Mandula, Phys. Rev. 159 (1967) 1251.

[7] R.Haag, J.Lopuszansky and M.Sohnius, Nucl.Phys. B88 (1975) 257.

[8] F.A. Berezin, The Method of Second Quantization, Moscow, Nauka, 1965.

[9] P. Fayet and J. Illiopoulos, Phys. Lett. B51 (1974) 461.

[10] L. O’Raifeartaigh, Nucl.Phys. B96 (1975) 331

[11] G. G. Ross, Grand Unified Theories, Benjamin \& Cummings, 1985.

[12] C. Amsler et al. (Particle Data Group), Phys. Lett. B667 (2008) 1.

[13] U. Amaldi, W. de Boer and H. Fürstenau, Phys. Lett. B260 (1991) 447.

[14] Y. Sofue, V. Rubin, Ann. Rev. Astron. Astrophys. 39 (2001) 137, and refs therein.

[15] C.S. Kochanek, Astrophys. J. 453 (1995) 545;

N.Kaiser, G.Squires, Astrophys. J. 404 (1993) 441.

[16] V.A. Ryabov, V.A. Tsarev and A.M. Tskhovrebov, Phys. Usp. 51 (2008) 1091, and refs therein.

[17] G. Jungman, M. Kamionkowski and K. Griest, Phys. Rep. 267 (1996) 195;

H. Goldberg, Phys. Rev. Lett. 50 (1983) 1419;

J.R. Ellis, et al., Nucl. Phys. B238 (1984) 453.

[18] M. Peskin and D. Schröder, An Introduction to Quantum Field Theory, Addison-Wesley, 1995.

[19] H. Baer and X. Tata, Weak Scale Supersymmetry, Cambridge University Press, 2006.

[20] H.E. Haber, Introductory Low-Energy Supersymmetry, Lectures given at TASI 1992, (SCIPP 92/33, 1993), hep-ph/9306207;

D.I. Kazakov, Beyond the Standard Model (In search of supersymmetry), Lectures at the ESHEP 2000, CERN-2001-003, hep-ph/ 0012288 ;

D. I. Kazakov, Beyond the Standard Model, Lectures at the ESHEP 2004, hep-ph/ 0411064 . D. Kazakov, Supersymmetry on the Run: LHC and Dark Matter, Nucl.Phys.Proc.Suppl. 203-204 (2010) 118Ü154, arXiv:1010.5419.

[21] A.V. Gladyshev, D.I. Kazakov, Supersymmetry and LHC, Phys. Atom. Nucl. 70 (2007) 1553, hep-ph / 0606288 . A.V. Gladyshev, D.I. Kazakov, Is (Low Energy) SUSY still alive? Conference: C12-06-06, e-Print: arXiv:1212.2548 Lectures at the European school on high energy physics 2012.

[22] P. Fayet, Nucl. Phys. B90 (1975) 104;

A. Salam and J. Srathdee, Nucl. Phys. B87 (1975) 85.

[23] U. Ellwanger, C. Hugonie, A.M. Teixeira, Phys. Rept. 496 (2010) 1, and refs therein; M. Maniatis, Int. J. Mod. Phys. A25 (2010) 3505, and refs therein.

[24] L. Hall, J. Lykken and S. Weinberg, Phys. Rev. D27 (1983) 2359; S.K. Soni and H.A. Weldon, Phys. Lett. B126 (1983) 215;

I. Affleck, M. Dine and N. Seiberg, Nucl. Phys. B256 (1985) 557. 
[25] H. P. Nilles, Phys. Lett. B115 (1982) 193;

A.H. Chamseddine, R. Arnowitt and P. Nath, Phys. Rev. Lett. 49 (1982) 970;

A.H. Chamseddine, R. Arnowitt and P. Nath, Nucl. Phys. B227 (1983) 121;

R. Barbieri, S. Ferrara and C. A. Savoy, Phys. Lett. B119 (1982) 343.

[26] M. Dine and A.E. Nelson, Phys. Rev. D48 (1993) 1277;

M. Dine, A.E. Nelson and Y. Shirman, Phys. Rev. D51 (1995) 1362.

[27] L. Randall and R. Sundrum, Nucl. Phys. B557 (1999) 79;

G.F. Giudice, M.A. Luty, H. Murayama and R. Rattazzi, JHEP, 9812 (1998) 027.

[28] D.E. Kaplan, G.D. Kribs and M. Schmaltz, Phys. Rev. D62 (2000) 035010;

Z. Chacko, M.A. Luty, A.E. Nelson and E. Ponton, JHEP, 0001 (2000) 003.

[29] G.G. Ross and R.G. Roberts, Nucl. Phys. B377 (1992) 571;

V. Barger, M.S. Berger and P. Ohmann, Phys. Rev. D47 (1993) 1093.

[30] W. de Boer, R. Ehret and D. Kazakov, Z. Phys. C67 (1995) 647;

W. de Boer et al., Z. Phys. C71 (1996) 415.

[31] L.E. Ibáñez, C. Lopéz and C. Muñoz, Nucl. Phys. B256 (1985) 218.

[32] V. Barger, M.S. Berger and P. Ohman, Phys. Rev. D49 (1994) 4908.

[33] V. Barger, M.S. Berger, P. Ohmann and R. Phillips, Phys. Lett. B314 (1993) 351;

P. Langacker and N. Polonsky, Phys. Rev. D49 (1994) 1454;

S. Kelley, J.L. Lopez and D.V. Nanopoulos, Phys. Lett. B274 (1992) 387.

[34] M. Carena, M. Quiros and C.E.M. Wagner, Nucl. Phys. B461 (1996) 407; A.V. Gladyshev, et al., Nucl. Phys. B498 (1997) 3;

A.V. Gladyshev, D.I. Kazakov, Mod. Phys. Lett. A10 (1995) 3129.

[35] S. Heinemeyer, W. Hollik and G. Weiglein, Phys. Lett. $B 455$ (1999) 179;

S. Heinemeyer, W. Hollik and G. Weiglein, Eur. Phys. J. C9 (1999) 343.

[36] A. Arbey, M. Battaglia, A. Djouadi, F. Mahmoudi, JHEP 1209 (2012) 107.

[37] A. Arbey, et al., Phys. Lett. B708 (2012) 162. 